\title{
Multi-stage Antenna Selection for Adaptive Beamforming in MIMO Arrays
}

\author{
Hamed Nosrati, Studnet Member, IEEE, Elias Aboutanios, Senior Member, IEEE, and David Smith, Member, IEEE
}

\begin{abstract}
Increasing the number of transmit and receive elements in multiple-input-multiple-output (MIMO) antenna arrays imposes a substantial increase in hardware and computational costs. We mitigate this problem by employing a reconfigurable MIMO array where large transmit and receive arrays are multiplexed in a smaller set of $k$ baseband signals. We consider four stages for the MIMO array configuration and propose four different selection strategies to offer dimensionality reduction in post-processing and achieve hardware cost reduction in digital signal processing (DSP) and radio-frequency (RF) stages. We define the problem as a determinant maximization and develop a unified formulation to decouple the joint problem and select antennas/elements in various stages in one integrated problem. We then analyze the performance of the proposed selection approaches and prove that, in terms of the output SINR, a joint transmit-receive selection method performs best followed by matched-filter, hybrid and factored selection methods. The theoretical results are validated numerically, demonstrating that all methods allow an excellent trade-off between performance and cost.
\end{abstract}

Index Terms-Antenna selection, MIMO radar, adaptive array beamforming, STAP, convex optimization.

\section{INTRODUCTION}

The spatial diversity and performance improvements offered by multiple-input multiple-output (MIMO) antenna systems have led to their widespread use in a variety of applications including wireless communications e.g. massive MIMO [1], [2], radar and sonar [3], [4]. In radar, MIMO arrays have proven effective at enhancing the radar's resolution as they offer increased number of degrees of freedom (DOFs) [5]. A MIMO phased array comprises an array of antennas, transmitting a set of noncoherent orthogonal waveforms that can be extracted at the receiver by a corresponding number of matched filters. Improved spatial diversity, parameter identifiability, and detection performance result from the added DoFs compared to single-input multiple-output (SIMO) configurations [3].

The advantages of the MIMO configuration are delivered at the expense of a significant increase in the problem dimensionality and hardware cost [6]. The system hardware include the antennas, baseband digital signal processing (DSP) and radio frequency $(\mathrm{RF})$ front-ends comprising the low noise amplifiers (LNA), phase shifters, and frequency mixers. Among these,

H. Nosrati is with the School of Electrical Engineering and Telecommunication, University of New South Wales, NSW 2052, Australia, and Data61, CSIRO (Commonwealth Scientific and Industrial Research Organisation), NSW 2015, Australia (e-mail:hamed.nosrati@unsw.edu.au).

E. Aboutanios is with the School of Electrical Engineering and Telecommunication, University of New South Wales, NSW 2052, Australia (email:elias@unsw.edu.au)

D. Smith, is with Data61, CSIRO (Commonwealth Scientific and Industrial Research Organisation), NSW 2015, Australia (email:david.smith@data61.csiro.au) the baseband DSP and RF front-ends are a great deal more expensive than the antenna elements. One way to reduce the cost while maintaining the spectral diversity is to employ a large antenna array but select a subset of antennas to feed through the RF switching network [7]-[9]. In this work, we focus on antenna selection in the context of MIMO radar.

Over the last decade, antenna selection in MIMO arrays has commanded significant attention both in wireless communications and radar applications. In communications, antenna selection is employed to maximize the channel capacity. To this end, near-optimal strategies that assume perfect knowledge of the channel were proposed in [10] and [11]. In [12], a fast adaptive antenna selection via discrete stochastic optimization in is proposed, where an aggressive stochastic approximation is employed to generate iteratively a sequence of estimates of the solution. More recently, antenna selection has been employed to reduce complexity and power consumption in $\mathrm{mm}$ wave MIMO systems through compressed spatial sampling of the received signal [13]. In radar, both deterministic and optimization-based methods have been developed to select a subset of antennas and reconfigure the array architecture in order to maximize the output signal-to-interference and noise ratio (SINR) [8], [9], [14], [15] and enhance the direction of arrival (DoA) estimation [16]. Antenna selection also plays an important role in aperture sharing in dual function radar communication systems [17], [18].

In MIMO radars, antenna selection has been studied mostly from the perspective of target parameter estimation. An optimal antenna placement was proposed in [19] to minimize the Cramér-Rao lower bound (CRLB) of the velocity estimates. In [20] a combinatorial optimization approach was used to achieve resource allocation for localization error minimization in multiple radar systems. The CRLB for target location in MIMO radars with collocated antennas was derived in [21], allowing its determinant to be minimized. Joint antenna subset selection and optimal power allocation were also implemented in [22] for localization in MIMO radar sensor networks via convex optimization. In a similar vein, the idea of minimum redundancy has been successfully applied to the design of physical transmit/receive arrays to form MIMO virtual arrays with maximum contiguous aperture, i.e. minimum redundancy virtual arrays (MRVA) [23]. The two-level autocorrelation property of the difference sets (DSs) was then successfully exploited to maximize the virtual aperture [24].

In this paper we address the problem of antenna selection for interference cancellation and SINR maximization. Antenna selection can be applied to the transmit and receive arrays separately, jointly to the transmit and receive arrays, or to the matched filter bank (virtual array). We study all of these 
scenarios and propose a comprehensive optimization method to derive their solutions. We first examine the joint transmit/recieve element selection, which reduces the dimensionality and consequently decreases the computational cost. We then consider the factored selection approach in which we separately select subsets of the transmit and receive arrays. We formulate the factored problem as a coupled optimization such that both selections are solved together. The computational cost of the MIMO radar can also be alleviated by reducing the number of matched filters used to generate a virtual array at the receiver, which involves the application of element selection to the virtual array. Finally, we bring these scenarios together in a hybrid selection strategy that is capable of reducing the number of transmitters, receivers and matched filters simultaneously in a unified approach.

The main contributions of this paper are as follows.

1) We express the output SINR, denoted as SINR out $_{\text {, as a }}$ function of selected elements of the MIMO array in a scenario comprising a single target, multiple jammers, and clutter.

2) We propose four different selection approaches, each achieving a different efficiency in terms of hardware (e.g., baseband and RF), computational, and power cost.

3) Since the SINR $_{\text {out }}$ is a joint function of transmitters and receivers in MIMO, we propose a new factored problem formulation that permits us to decouple the transmit and receive sides and allows their designs to be performed separately.

4) We formulate the dual problem and study the performance of the proposed selection methods from a mathematical point of view.

5) We propose a relaxation method and successfully approximate the global solution via a set of problemspecific randomized rounding strategies.

The rest of this paper is organized as follows. In section II we present the formulation of the SINR $_{\text {out }}$ maximization using element selection. We then study the selection approaches in Section III. The relaxation strategy is detailed in Section IV. and the numerical results are presented in Section $\mathrm{V}$. Finally some conclusions are drawn in section VI

\section{Notation}

We use bold lower-case letters to denote vectors, and uppercase letters for matrices. The notation $\mathbb{E}$ is the expectation operator, and $\operatorname{Tr}(\mathbf{M})$ denotes the trace of $\mathbf{M} .(\bullet)^{T}$ and $(\bullet)^{H}$ are the Hermitian and transpose operations. The operation $\operatorname{diag}(\mathbf{v})$ constructs a square diagonal with $\mathbf{v}$ along the diagonal, whereas $\operatorname{diag}(\mathbf{M})$ extracts the diagonal of $\mathbf{M}$. The function real $(\bullet)$ takes the real part of its complex argument. We use $\otimes$ for Kronecker product. Finally, $\mathbf{1}_{N}$ is a $N \times 1$ vector of all ones, $\mathbf{0}_{N}$ a vector of zeros, and $\mathbf{I}_{N}$ the $N \times N$ identity matrix.

\section{PRoBlem Formulation}

Let us consider a MIMO radar equipped with $M$ transmitters and $N$ receivers as shown in Fig. 1. Each transmitter emits one of the predesigned orthogonal waveforms from

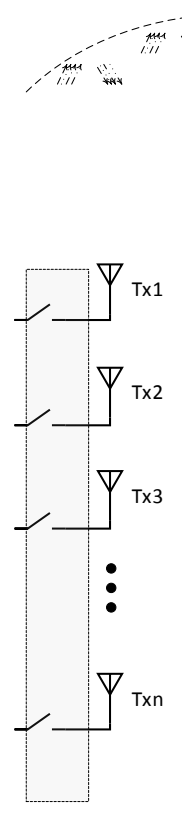

1

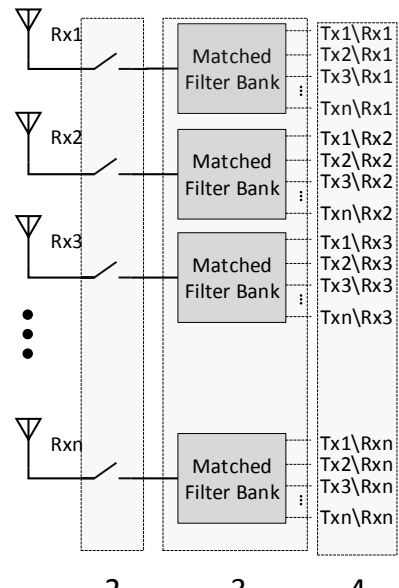

2

4
Fig. 1. Block diagram of the considered MIMO system. Stage 1: transmit antenna selection. Stage 2: receive antenna selection. Stage 3: matched-filter selection in DSP. Stage 4: output signal selection.

the waveform vector $\phi(t)=\left[\phi_{1}(t), \phi_{2}(t), \ldots, \phi_{M}(t)\right]$. The snapshot vector received by the receive array for pulse $\tau$ is

$$
\mathbf{x}(t, \tau)=\mathbf{x}_{\mathrm{s}}(t, \tau)+\mathbf{x}_{\mathrm{c}}(t, \tau)+\mathbf{x}_{\mathrm{j}}(t, \tau)+\mathbf{x}_{\mathrm{n}}(t, \tau),
$$

where $\mathbf{x}_{c}$ and $\mathbf{x}_{j}$ represent the clutter and jammer respectively. The signal of interest (SOI), $\mathbf{x}_{s}$, represents the target reflection and $\mathbf{x}_{n}$ is zero-mean additive Gaussian noise with variance $\sigma_{n}^{2}$. By applying matched-filtering with respect to the $M$ orthogonal waveforms, the extended receive signal becomes

$$
\begin{aligned}
\tilde{\boldsymbol{x}}(\tau) & =\operatorname{vec}\left(\int_{T_{p}} \mathbf{x}(t, \tau) \boldsymbol{\phi}^{H}(t) d t\right) \\
& =\tilde{\boldsymbol{x}}_{\mathrm{s}}(\tau)+\tilde{\boldsymbol{x}}_{\mathrm{c}}(\tau)+\tilde{\boldsymbol{x}}_{\mathrm{j}}(\tau)+\tilde{\boldsymbol{x}}_{\mathrm{n}}(\tau)
\end{aligned}
$$

where $\tilde{\boldsymbol{x}}$ represents the vectorized version of the matchedfiltered snapshot vector. Exploiting the orthogonality assumption, we can write the SOI as

$$
\begin{aligned}
\tilde{\boldsymbol{x}}_{\mathrm{s}}(\tau) & =\beta_{s} \mathbf{a}_{\mathrm{s}} \\
\mathbf{a}_{\mathrm{s}} & =\mathbf{a}_{\mathrm{t}}\left(\theta_{\mathrm{t}}\right) \otimes \mathbf{a}_{\mathrm{r}}\left(\theta_{\mathrm{r}}\right),
\end{aligned}
$$

where $\beta_{\mathrm{s}}$ is the target reflection coefficient, which we assume obeys the Swirling II model. The transmit steering vector, $\mathbf{a}_{\mathrm{t}}\left(\theta_{\mathrm{t}}\right)$, corresponds the Direction-of-Departure (DoD) $\theta_{\mathrm{t}}$, and the receive steering vector, $\mathbf{a}_{\mathrm{r}}\left(\theta_{\mathrm{r}}\right)$, is associated with DoA $\theta_{\mathrm{r}}$. In the case of a ULA, the steering vectors are given by

$$
\begin{aligned}
& \mathbf{a}_{\mathrm{t}}\left(\theta_{\mathrm{t}}\right)=\left[1, e^{j 2 \pi d_{\mathrm{t}} \sin \theta_{\mathrm{t}}}, e^{j 2 \pi 2 d_{\mathrm{t}} \sin \theta_{\mathrm{t}}}, \ldots, e^{j 2 \pi M d_{\mathrm{t}} \sin \theta_{\mathrm{t}}}\right], \\
& \mathbf{a}_{\mathrm{r}}\left(\theta_{\mathrm{r}}\right)=\left[1, e^{j 2 \pi d_{\mathrm{r}} \sin \theta_{\mathrm{r}}}, e^{j 2 \pi 2 d_{\mathrm{r}} \sin \theta_{\mathrm{r}}}, \ldots, e^{j 2 \pi N d_{\mathrm{r}} \sin \theta_{\mathrm{r}}}\right],
\end{aligned}
$$

with $d_{\mathrm{t}}, d_{\mathrm{r}}$ denoting the inter-element spacing employed in transmit and receive arrays. 
Now assuming a set of angle cells, $\left\{\theta_{i}\right\}_{i=1}^{N_{\mathrm{c}}}$, we model the clutter as the reflections from these directions and extract the received clutter signal as

$$
\begin{aligned}
\tilde{\boldsymbol{x}}_{\mathrm{c}}(\tau) & =\sum_{i=1}^{N_{\mathrm{c}}} \beta_{i} \mathbf{a}_{\mathrm{c}, i} \\
\mathbf{a}_{\mathrm{c}, i} & =\mathbf{a}_{\mathrm{t}}\left(\theta_{\mathrm{tc}, i}\right) \otimes \mathbf{a}_{\mathrm{r}}\left(\theta_{\mathrm{rc}, i}\right),
\end{aligned}
$$

where $\beta_{i}$ is the reflection coefficient of the $i$-th clutter cell from directions $\theta_{\mathrm{tc}, i}$, and $\theta_{\mathrm{rc}, i}$ with respect to transmit, and receive sides. Suppose that $N_{\mathrm{j}}$ jamming signals are in the field of view of the radar. Then the jamming signal is expressed as

$$
\begin{aligned}
\tilde{\boldsymbol{x}}_{\mathrm{j}}(\tau) & =\sum_{i=1}^{N_{\mathrm{j}}} \alpha_{i} \mathbf{a}_{\mathrm{j}, i} \\
\mathbf{a}_{\mathrm{j}, i} & =\tilde{\boldsymbol{x}}_{\mathrm{j}, \mathrm{i}}(\tau) \otimes \mathbf{a}_{\mathrm{r}}\left(\theta_{\mathrm{rj}, i}\right),
\end{aligned}
$$

with $\alpha_{i}$, and $\tilde{\boldsymbol{x}}_{\mathrm{j}, \mathrm{i}}(\tau)$ denoting the complex amplitude, and the matched filtered version of the $i$-th jamming signal. Given a strong jamming source $\mathbf{x}_{\mathrm{j}, \mathrm{i}}(t, \tau)$ with a power of $\hat{\alpha}_{i}$, which emulates the radar orthogonal waveforms we have

$$
\mathbf{x}_{\mathrm{j}, \mathrm{i}}(t, \tau)=\hat{\alpha_{i}} \sum_{i=1}^{M} \phi_{i}(t, \tau)
$$

then, this signal passes through the matched filters and by incorporating $\hat{\alpha}_{i}$ in $\alpha_{i}$ we can write $\tilde{\boldsymbol{x}}_{\mathrm{j}, \mathrm{i}}(\tau)=\mathbf{1}$ [25], [26]. The received signal is then input to an adaptive filter with weights vector, $\mathbf{w}$, giving the output

$$
y(\tau)=\mathbf{w}^{H} \tilde{\boldsymbol{x}}(\tau) .
$$

The weights vector that preserves the SOI, $\tilde{\boldsymbol{x}}_{\mathrm{s}}$, while suppressing the clutter, jammers and noise, thus maximizing the output SINR, is obtained by solving the following optimization

$$
\begin{aligned}
& \min _{\mathbf{w}} \mathbf{w}^{H} \mathbf{R w} \\
& \text { s.t. } \mathbf{w}^{H} \mathbf{a}_{\mathrm{s}}=1 .
\end{aligned}
$$

This yields the solution [27]

$$
\mathbf{w}=\frac{\mathbf{R}^{-1} \mathbf{a}_{\mathrm{s}}}{\mathbf{a}_{\mathrm{s}}^{H} \mathbf{R}^{-1} \mathbf{a}_{\mathrm{s}}},
$$

where $\mathbf{R}$ is the interference (jamming and clutter) plus noise covariance matrix of size $M N \times M N$. Assuming the clutter, jammers and noise are statistically independent, we have

$$
\mathbf{R}=\mathbf{R}_{\mathrm{c}}+\mathbf{R}_{\mathrm{j}}+\mathbf{R}_{n} .
$$

Taking the clutter scattering coefficients, $\beta_{i}$ to be mutually uncorrelated, we find that

$$
\mathbf{R}_{\mathrm{c}}=\mathbb{E}\left\{\tilde{\boldsymbol{x}}_{\mathrm{c}}(\tau) \tilde{\boldsymbol{x}}_{\mathrm{c}}^{H}(\tau)\right\}=\sum_{i=1}^{N_{\mathrm{c}}} \sigma_{\mathrm{c}, i}^{2} \mathbf{a}_{\mathrm{c}, i} \mathbf{a}_{\mathrm{c}, i}^{H}=\mathbf{A}_{\mathrm{c}} \mathbf{P}_{\mathrm{c}} \mathbf{A}_{\mathrm{c}}^{H},
$$

where

$$
\begin{aligned}
\sigma_{\mathrm{c}, i}^{2} & =\mathbb{E}\left\{\beta_{i} \beta_{i}^{*}\right\}, \\
\mathbf{A}_{\mathrm{c}} & =\left[a_{\mathrm{c}, 1}, a_{\mathrm{c}, 2}, \ldots, a_{\mathrm{c}, N_{\mathrm{c}}}\right], \quad M N \times N_{\mathrm{c}}
\end{aligned}
$$

and

$$
\mathbf{P}_{\mathrm{c}}=\operatorname{diag}\left(\sigma_{c, 1}^{2}, \ldots, \sigma_{c, N_{\mathrm{c}}}^{2}\right) .
$$

Similarly, using (1), the covariance matrix for the jamming signal is found to be

$$
\mathbf{R}_{\mathrm{j}}=\mathbb{E}\left\{\tilde{\boldsymbol{x}}_{\mathrm{j}}(\tau) \tilde{\boldsymbol{x}}_{\mathrm{j}}^{H}(\tau)\right\}=\mathbf{A}_{\mathbf{j}} \mathbf{P}_{\mathrm{j}} \mathbf{A}_{\mathrm{j}}^{H},
$$

where $\mathbf{A}_{\mathrm{j}}$, and $\mathbf{P}_{\mathrm{j}}$ are $M N \times M N_{\mathrm{j}}$, and $M N_{\mathrm{j}} \times M N_{\mathrm{j}}$ matrices denoted as

$$
\begin{aligned}
& \mathbf{A}_{\mathrm{j}}=[\underbrace{\mathbf{a}_{\mathrm{r}}\left(\theta_{\mathrm{rj}, 1}\right), \ldots, \mathbf{a}_{\mathrm{r}}\left(\theta_{\mathrm{rj}, 1}\right)}_{M \text { times }}, \ldots, \underbrace{\mathbf{a}_{\mathrm{r}}\left(\theta_{\mathrm{rj}, N_{\mathrm{j}}}\right), \ldots, \mathbf{a}_{\mathrm{r}}\left(\theta_{\mathrm{rj}, N_{\mathrm{j}}}\right)}_{M \text { times }}], \\
& \mathbf{P}_{\mathrm{j}}=\operatorname{diag}(\underbrace{\sigma_{j, 1}^{2}, \ldots, \sigma_{j, 1}^{2}}_{M \text { times }}, \ldots, \underbrace{\sigma_{j, N_{\mathrm{j}}}^{2}, \ldots, \sigma_{j, N_{\mathrm{j}}}^{2}}_{M \text { times }}),
\end{aligned}
$$

and

$$
\sigma_{\mathrm{j}, i}^{2}=\mathbb{E}\left\{\alpha_{i} \alpha_{i}^{*}\right\}
$$

Finally, the covariance matrix of the white noise is given by

$$
\mathbf{R}_{n}=\mathbb{E}\left\{\tilde{\boldsymbol{x}}_{\mathrm{n}}(\tau) \tilde{\boldsymbol{x}}_{\mathrm{n}}^{H}(\tau)\right\}=\sigma_{n}^{2} \mathbf{I}_{M N} .
$$

Now writing $\sigma_{\mathrm{s}}^{2}=\mathbb{E}\left\{\beta_{\mathrm{s}} \beta_{\mathrm{s}}^{*}\right\}$, the SINR at the output of the filter (2) is

$$
\mathrm{SINR}_{\text {out }}=\sigma_{\mathrm{s}}^{2} \mathbf{a}_{\mathrm{s}}^{H} \mathbf{R}^{-1} \mathbf{a}_{\mathrm{s}} .
$$

The inverse covariance matrix becomes

$$
\begin{aligned}
\mathbf{R}^{-1} & =\left(\mathbf{A}_{\mathrm{c}} \mathbf{P}_{\mathrm{c}} \mathbf{A}_{\mathrm{c}}^{H}+\mathbf{A}_{\mathrm{j}} \mathbf{P}_{\mathrm{j}} \mathbf{A}_{\mathrm{j}}^{H}+\sigma_{n}^{2} \mathbf{I}_{M N}\right)^{-1} \\
& \stackrel{(\mathrm{a})}{=}\left(\mathbf{A}_{\mathrm{jc}} \mathbf{P}_{\mathrm{jc}} \mathbf{A}_{\mathrm{jc}}^{H}+\sigma_{n}^{2} \mathbf{I}_{M N}\right)^{-1} \\
& \stackrel{(\mathrm{b})}{=} \sigma_{n}^{-2}\left(I_{M N}-\mathbf{A}_{\mathrm{jc}}\left(\sigma_{n}^{2} \mathbf{P}_{\mathrm{jc}}^{-1}+\mathbf{A}_{\mathrm{jc}}^{H} \mathbf{A}_{\mathrm{jc}}\right)^{-1} \mathbf{A}_{\mathrm{jc}}^{H}\right),
\end{aligned}
$$

where in (a) we put $\mathbf{A}_{\mathrm{jc}}=\left[\mathbf{A}_{\mathrm{c}}, \mathbf{A}_{\mathrm{j}}\right]$, and $\mathbf{P}_{\mathrm{jc}}=\operatorname{diag}\left(\mathbf{P}_{\mathrm{c}}, \mathbf{P}_{\mathrm{j}}\right)$, and (b) follows from the matrix inversion lemma. Now, we can reformulate the output SINR as

$$
\operatorname{SINR}_{\text {out }}=\frac{\sigma_{s}^{2}}{\sigma_{n}^{2}} \mathbf{a}_{s}^{H}\left(\mathbf{I}_{M N}-\mathbf{A}_{\mathrm{jc}}\left(\sigma_{n}^{2} \mathbf{P}_{\mathrm{jc}}^{-1}+\mathbf{A}_{\mathrm{jc}}^{H} \mathbf{A}_{\mathrm{jc}}\right)^{-1} \mathbf{A}_{\mathrm{jc}}^{H}\right) \mathbf{a}_{s} .
$$

Defining the matrices

$$
\mathbf{B}_{\mathrm{jc}}=\sigma_{n}^{2} \mathbf{P}_{\mathrm{jc}}^{-1}, \quad \mathbf{A}_{\mathrm{s}}=\left[\mathbf{a}_{s}, \mathbf{A}_{\mathrm{jc}}\right], \quad \mathbf{B}_{\mathrm{s}}=\left[\mathbf{0}, \sigma_{n}^{2} \mathbf{P}_{\mathrm{jc}}^{-1}\right],
$$

and making use of the determinant formula of block matrices, we obtain [17], [28]

$$
\begin{aligned}
& \left|\mathbf{A}_{\mathrm{s}}^{H} \mathbf{A}_{\mathrm{s}}+\mathbf{B}_{\mathrm{s}}\right|=\left|\begin{array}{cc}
\mathbf{a}_{s}^{H} \mathbf{a}_{s} & \mathbf{a}_{s}^{H} \mathbf{A}_{\mathrm{jc}} \\
\mathbf{A}_{\mathrm{jc}}^{H} \mathbf{a}_{s} & \mathbf{A}_{\mathrm{jc}}^{H} \mathbf{A}_{\mathrm{jc}}+\mathbf{B}_{\mathrm{jc}}
\end{array}\right| \\
& =\left|\mathbf{A}_{\mathrm{jc}}^{H} \mathbf{A}_{\mathrm{jc}}+\mathbf{B}_{\mathrm{jc}}\right| \\
& \times \mathbf{a}_{s}^{H}\left(\mathbf{I}_{M N}-\mathbf{A}_{\mathrm{jc}}\left(\mathbf{B}_{\mathrm{jc}}+\mathbf{A}_{\mathrm{jc}}^{H} \mathbf{A}_{\mathrm{jc}}\right)^{-1} \mathbf{A}_{\mathrm{jc}}^{H}\right) \mathbf{a}_{s} .
\end{aligned}
$$

Substituting this into the expression of SINR $_{\text {out }}$ yields

$$
\operatorname{SINR}_{\text {out }}=\frac{\sigma_{s}^{2}}{\sigma_{n}^{2}} \frac{\left|\mathbf{A}_{\mathrm{s}}^{H} \mathbf{A}_{\mathrm{s}}+\mathbf{B}_{\mathrm{s}}\right|}{\left|\mathbf{A}_{\mathrm{jc}}^{H} \mathbf{A}_{\mathrm{jc}}+\mathbf{B}_{\mathrm{jc}}\right|},
$$

where |.| denotes the determinant and the signal-to-noise ratio, $\mathrm{SNR}=\frac{\sigma_{s}^{2}}{\sigma_{n}^{2}}$. This reveals that, although the SNR is constant, the set of active transmit and receive elements directly affects the achieved output SINR by varying the interplay among the jamming and clutter steering vectors and their powers. 
Let us introduce a binary vector $\mathbf{c}$ with elements 0 if their corresponding elements are inactive and 1 otherwise. Then the element selection can be incorporated into the expression of the output SINR as follows

$$
\operatorname{SINR}_{\text {out }}(\mathbf{c})=\frac{\sigma_{s}^{2}}{\sigma_{n}^{2}} h(\mathbf{c}),
$$

where

$$
h(\mathbf{c})=\frac{\left|\mathbf{A}_{\mathrm{s}}^{H} \operatorname{diag}(\mathbf{c}) \mathbf{A}_{\mathrm{s}}+\mathbf{B}_{\mathrm{s}}\right|}{\left|\mathbf{A}_{\mathrm{jc}}^{H} \operatorname{diag}(\mathbf{c}) \mathbf{A}_{\mathrm{jc}}+\mathbf{B}_{\mathrm{jc}}\right|} .
$$

The optimum set of $k$ active elements that maximizes the SINR is found via the following optimization:

$$
\begin{aligned}
\max _{\mathbf{c}} & \operatorname{SINR}_{\mathrm{out}}(\mathbf{c}) \\
\text { s.t. } & c_{i}^{2}-c_{i}=0 \quad i=1 \ldots M N, \\
& \mathbf{c}^{T} \mathbf{c}=k .
\end{aligned}
$$

The optimization in (3) is maximization of the volume of two ellipsoids. Therefore, we may employ log-determinant function as

$$
\begin{aligned}
f(\mathbf{c})= & \log \operatorname{det}(h(\mathbf{c})) \\
= & \log \operatorname{det}\left(\mathbf{A}_{\mathrm{s}}^{H} \operatorname{diag}(\mathbf{c}) \mathbf{A}_{\mathrm{s}}+\mathbf{B}_{\mathrm{s}}\right) \\
& -\log \operatorname{det}\left(\mathbf{A}_{\mathrm{jc}}^{H} \operatorname{diag}(\mathbf{c}) \mathbf{A}_{\mathrm{jc}}+\mathbf{B}_{\mathrm{jc}}\right) .
\end{aligned}
$$

This problem can be effectively solved via a log-determinant relaxation and a sequential convex programming (SCP) procedure accordingly. We will elaborate on the solution approximation in Section IV

Considering (3), we propose four different methods to apply selection in a MIMO radar. We list all the requirements in different modes in a quadratic form. Hence, we cast the general problem of antenna selection in a MIMO radar as follows

$$
\begin{array}{rl}
\max _{\mathbf{c}} & f(\mathbf{c}) \\
& \mathbf{c}^{T} \mathbf{W}_{i} \mathbf{c}+\mathbf{q}_{i}^{T} \mathbf{c}+r_{i} \leq 0, \quad i=1, \ldots, \ell .
\end{array}
$$

where $\mathbf{W}_{i} \in \mathbb{S}^{M N}, \mathbf{q}_{i} \in \mathbb{R}^{M N}$, and $r_{i} \in \mathbb{R}$. Note that $\mathbb{S}^{n}$ denotes the set of $n \times n$ symmetric matrices, and $\mathbb{R}^{n}$ represents the set of real vectors of size $n$.

\section{Selection StRATEGies}

The selection strategy may be applied at each of the four stages of a MIMO radar depicted in Fig. 1. In what follows, we detail and compare these selection approaches.

\section{A. Joint Tx-Rx selection}

The first selection strategy involves thinning the MIMO virtual array by selecting the individual matched-filters at the output (stage 4) of Fig. 1 In this case, we reshape the selection vector $\mathbf{c}$ as a matrix $\mathbf{C}$

$$
\mathbf{C}=\begin{aligned}
& \operatorname{Tx}_{1} \\
& \operatorname{Rx}_{1} \\
& \vdots \\
& \operatorname{Rx}_{2} \\
& \operatorname{Rx}_{N}
\end{aligned}\left(\begin{array}{cccc}
c_{1,1} & c_{1,2} & \cdots & c_{1, M} \\
c_{2,1} & c_{2,2} & \cdots & c_{2, M} \\
\vdots & \vdots & \ddots & \vdots \\
c_{N, 1} & c_{N, 2} & \cdots & c_{N, M}
\end{array}\right),
$$

such that $\mathbf{c}=\operatorname{vec}(\mathbf{C})$, and $c_{i, j}$ is the entry that indicates whether the $j$-th matched filter (which extracts the $j$-th waveform) in the $i$-th receiver is selected. The joint thinning mode is obtained by directly selecting $k$ elements in c. The selection problem of (4) can now be written as

$$
\begin{array}{rl}
\max _{\mathbf{c}} & f(\mathbf{c}) \\
\text { s.t. } & c_{i}^{2}-c_{i}=0 \quad i=1 \ldots M N, \\
& \mathbf{c}^{T} \mathbf{c}=k .
\end{array}
$$

In this formulation, we place a constraint only on the number of output signals and any subset of the output matched filters is a possible solution. The joint selection finds the best subset, which decreases the dimensionality of the signal used in the post processing (e.g. for detection, estimation or other tasks). However, the entire system including transmitters, receivers, and matched filters should be active, and consequently the hardware cost and power consumption remain high.

\section{B. Factored Tx and Rx selection}

As all of the transmitters are required to be active in the joint selection strategy, transmitters that do not contribute to the selected set of matched-filters effectively waste the power alloted to them. This issue can be mitigated by factorizing the selection problem into transmit and receive sub-problems. Suppose that we select $k_{\mathrm{t}}$ out of $M$ transmitting antennas (stage 1 in Fig. 1) and $k_{\mathrm{r}}$ out of the available $N$ receive antennas (stage 2 in Fig. 1). In terms of the selection matrix (5), this strategy selects $k_{\mathrm{r}}$ rows and $k_{\mathrm{t}}$ columns. Then the factored selection involves the optimization of two selection vectors jointly, one for the transmitters and the other for the receivers. We now develop a novel way to reformulate this coupled problem in one unified formulation.

Let $\mathbf{V}_{\mathrm{t}}=\left\{\mathbf{v}_{\mathrm{t}, 1}, \ldots, \mathbf{v}_{\mathrm{t}, M}\right\}$, and $\mathbf{V}_{\mathrm{r}}=\left\{\mathbf{v}_{\mathrm{r}, 1}, \ldots, \mathbf{v}_{\mathrm{r}, N}\right\}$ be a set of binary vectors each of which denoting a specific transmitter or receiver in the selection matrix

$$
\begin{aligned}
& \mathbf{v}_{\mathrm{t}, i}=\operatorname{vec}\left(\left[\mathbf{0}, \ldots, \underset{\operatorname{Tx}_{i}}{\mathbf{1}}, \ldots, \mathbf{0}\right]\right) \\
& \mathbf{v}_{\mathrm{r}, i}=\operatorname{vec}\left(\left[\mathbf{0}, \ldots, \underset{\mathbf{R x}_{i}}{\mathbf{1}}, \ldots, \mathbf{0}\right]^{T}\right)
\end{aligned}
$$


Then, the factored selection problem may be expressed as

$$
\begin{array}{rl}
\max _{\mathbf{c}} & f(\mathbf{c}) \\
\text { s.t. } & c_{i}^{2}-c_{i}=0 \quad i=1 \ldots M N \\
& \mathbf{c}^{T} \mathbf{P}_{\mathrm{t}, i} \mathbf{c} \in\left\{0, k_{\mathrm{r}}\right\} \quad i=1 \ldots M \\
& \mathbf{c}^{T} \mathbf{P}_{\mathrm{r}, i} \mathbf{c} \in\left\{0, k_{\mathrm{t}}\right\} \quad i=1 \ldots N \\
& \mathbf{c}^{T} \mathbf{c}=k_{\mathrm{t}} k_{\mathrm{r}}
\end{array}
$$

where

$\mathbf{P}_{\mathrm{t}, i}=\operatorname{diag}\left(\mathbf{v}_{\mathrm{t}, i}\right), i=1, \ldots, M, \mathbf{P}_{\mathrm{r}, i}=\operatorname{diag}\left(\mathbf{v}_{\mathrm{r}, i}\right), i=1, \ldots, N$.

In $6 b$ and $6 c$ we constrain the number of active elements in each column (row) to be exactly 0 or $k_{\mathrm{t}}\left(0\right.$ or $k_{\mathrm{r}}$ ).

Now let us define $\mathbf{Q}$ as the rectangular matrix

$$
\mathbf{Q}=\mathbf{P P}^{T}, \text { where } \mathbf{P}=\left[\mathbf{v}_{\mathrm{t}, 1}, \ldots, \mathbf{v}_{\mathrm{t}, M}, \mathbf{v}_{\mathrm{r}, 1}, \ldots, \mathbf{v}_{\mathrm{r}, N}\right] .
$$

Theorem 1: Let $\mathcal{S}$ be the set of selection vectors in conjunction with a factored selection problem comprising of $k_{\mathrm{t}}$ and $k_{\mathrm{r}}$ out of $M$ transmitters and $N$ receivers respectively. Then $\mathcal{S}$ is given by

$$
\mathcal{S}=\mathcal{S}_{1} \cap \mathcal{S}_{2} \cap \mathcal{S}_{3} \cap \mathcal{S}_{4},
$$

where the sets $\mathcal{S}_{1}-\mathcal{S}_{4}$ are defined as

$$
\begin{aligned}
& \mathcal{S}_{1}=\left\{\mathbf{c} \mid \mathbf{c}^{T} \mathbf{Q} \mathbf{c}=k_{\mathrm{r}} k_{\mathrm{t}}\left(k_{\mathrm{r}}+k_{\mathrm{t}}\right)\right\} \\
& \mathcal{S}_{2}=\left\{\mathbf{c} \mid \mathbf{c}^{T} \mathbf{P}_{\mathrm{t}, i} \mathbf{c} \leq k_{\mathrm{r}} \quad i=1 \ldots M,\right\} \\
& \mathcal{S}_{3}=\left\{\mathbf{c} \mid \mathbf{c}^{T} \mathbf{P}_{\mathrm{r}, i} \mathbf{c} \leq k_{\mathrm{t}} \quad i=1 \ldots N\right\} \\
& \mathcal{S}_{4}=\left\{\mathbf{c} \mid \mathbf{c}^{T} \mathbf{c}=k_{\mathrm{t}} k_{\mathrm{r}}\right\} .
\end{aligned}
$$

Proof: See Appendix A

Like the constraint in (6a), the binary constraints involving the quadratic forms in (6b) and 6c are non-convex. Therefore, we propose relaxing them by employing the following set of quadratic constraints instead

$$
\begin{gathered}
\mathbf{c}^{T} \mathbf{Q} \mathbf{c}=k_{\mathrm{r}} k_{\mathrm{t}}\left(k_{\mathrm{r}}+k_{\mathrm{t}}\right) \\
\mathbf{c}^{T} \mathbf{P}_{\mathrm{t}, i} \mathbf{c} \leq k_{\mathrm{r}} \quad i=1 \ldots M \\
\mathbf{c}^{T} \mathbf{P}_{\mathrm{r}, i} \mathbf{c} \leq k_{\mathrm{t}} \quad i=1 \ldots N,
\end{gathered}
$$

Using Theorem 11, the factored selection problem becomes

$$
\begin{array}{cl}
\max _{\mathbf{c}} & f(\mathbf{c}) \\
\text { s.t. } & c_{i}\left(c_{i}-1\right)=0 \quad i=1 \ldots M N, \\
& \mathbf{c}^{T} \mathbf{c}=k_{\mathrm{t}} k_{\mathrm{r}} \\
& \mathbf{c}^{T} \mathbf{Q} \mathbf{c}=k_{\mathrm{r}} k_{\mathrm{t}}\left(k_{\mathrm{r}}+k_{\mathrm{t}}\right) \\
& \mathbf{c}^{T} \mathbf{P}_{\mathrm{t}, i} \mathbf{c} \leq k_{\mathrm{r}} \quad i=1 \ldots M \\
& \mathbf{c}^{T} \mathbf{P}_{\mathrm{r}, i} \mathbf{c} \leq k_{\mathrm{t}} \quad i=1 \ldots N
\end{array}
$$

We recast the added binary constraints in the factored problem, into a quadratic form as a special case of (4). This enables us to compare the performance of the factored selection with that of the joint selection. We show that the optimum solution (i.e. SINR) obtained by the Lagrange dual of the joint selection optimization is always greater than or equal to that yielded by the factored problem. To this end, we derive a dual problem for the factored selection problem in
(7). We revise the factored problem (7), by introducing new variables $\mathbf{X}$, and $\mathbf{Y}$ as

$$
\begin{aligned}
\underset{\mathbf{c}}{\min } \log \operatorname{det}\left(\mathbf{X}^{-1}\right)-\log \operatorname{det}\left(\mathbf{Y}^{-1}\right) \\
\text { s.t. } \\
\boldsymbol{\Lambda}: \mathbf{X}=\mathbf{A}_{\mathrm{s}}^{H} \operatorname{diag}(\mathbf{c}) \mathbf{A}_{\mathrm{s}}+\mathbf{B}_{\mathrm{s}} \\
\boldsymbol{\Delta}: \mathbf{Y}=\mathbf{A}_{\mathrm{jc}}^{H} \operatorname{diag}(\mathbf{c}) \mathbf{A}_{\mathrm{jc}}+\mathbf{B}_{\mathrm{jc}} \\
\mu_{i}: \quad c_{i}\left(c_{i}-1\right)=0 \quad i=1 \ldots M N, \\
\nu: \mathbf{c}^{T} \mathbf{c}=k_{\mathrm{t}} k_{\mathrm{r}} \\
\lambda: \mathbf{c}^{T} \mathbf{Q} \mathbf{c} \leq k_{\mathrm{r}} k_{\mathrm{t}}\left(k_{\mathrm{r}}+k_{\mathrm{t}}\right) \\
\rho_{i}: \mathbf{c}^{T} \mathbf{P}_{\mathrm{t}, i} \mathbf{c} \leq k_{\mathrm{r}} \quad i=1 \ldots M, \\
\eta_{i}: \mathbf{c}^{T} \mathbf{P}_{\mathrm{r}, i} \mathbf{c} \leq k_{\mathrm{t}} \quad i=1 \ldots N,
\end{aligned}
$$

with Lagrange multipliers $\boldsymbol{\Lambda} \in \mathbb{S}^{n+1}\left(n=N_{\mathrm{c}}+N_{\mathrm{j}}\right), \boldsymbol{\Delta} \in \mathbb{S}^{n}$, $\boldsymbol{\mu} \in \mathbb{R}^{M N}, \boldsymbol{\rho} \in \mathbb{R}^{M}, \boldsymbol{\eta} \in \mathbb{R}^{N}$, and $\nu, \lambda \in \mathbb{R}$. We then introduce the Lagrangian

$$
\begin{aligned}
& L^{\mathrm{fct}}(\mathbf{c}, \mathbf{X}, \mathbf{Y}, \boldsymbol{\Lambda}, \boldsymbol{\Delta}, \boldsymbol{\mu}, \nu, \lambda, \boldsymbol{\rho}, \boldsymbol{\eta})= \\
& \quad \log \operatorname{det}\left(\mathbf{X}^{-1}\right)-\log \operatorname{det}\left(\mathbf{Y}^{-1}\right)+\operatorname{tr}(\mathbf{X} \boldsymbol{\Lambda})+\operatorname{tr}(\mathbf{Y} \boldsymbol{\Delta}) \\
& \quad+\mathbf{c}^{T}\left(\operatorname{diag}(\boldsymbol{\mu})+\nu \mathbf{I}+\lambda \mathbf{Q}+\sum_{i=1}^{M} \rho_{i} \mathbf{P}_{t, i}+\sum_{i=1}^{N} \eta_{i} \mathbf{P}_{r, i}\right) \mathbf{c} \\
& \quad-\boldsymbol{\Lambda}\left(\mathbf{A}_{\mathrm{s}}^{H} \operatorname{diag}(\mathbf{c}) \mathbf{A}_{\mathrm{s}}+\mathbf{B}_{\mathrm{s}}\right)-\boldsymbol{\Delta}\left(\mathbf{A}_{\mathrm{jc}}^{H} \operatorname{diag}(\mathbf{c}) \mathbf{A}_{\mathrm{jc}}+\mathbf{B}_{\mathrm{jc}}\right) \\
& \quad-\boldsymbol{\mu}^{T} \mathbf{c}-k_{\mathrm{t}} k_{\mathrm{r}} \nu-\lambda k_{\mathrm{r}} k_{\mathrm{t}}\left(k_{\mathrm{r}}+k_{\mathrm{t}}\right) \\
& \quad-k_{\mathrm{r}} \sum_{i=1}^{M} \rho_{i}-k_{\mathrm{t}} \sum_{i=1}^{N} \eta_{i} .
\end{aligned}
$$

By rearranging the Lagrangian we get

$$
\begin{aligned}
& L^{\mathrm{fct}}(\mathbf{c}, \mathbf{X}, \mathbf{Y}, \boldsymbol{\Lambda}, \boldsymbol{\Delta}, \boldsymbol{\mu}, \nu, \lambda, \boldsymbol{\rho}, \boldsymbol{\eta})= \\
& \quad \log \operatorname{det}\left(\mathbf{X}^{-1}\right)-\log \operatorname{det}\left(\mathbf{Y}^{-1}\right)+\operatorname{tr}(\mathbf{X} \boldsymbol{\Lambda})+\operatorname{tr}(\mathbf{Y} \boldsymbol{\Delta}) \\
& \quad-\operatorname{tr}\left(\mathbf{B}_{\mathrm{s}} \boldsymbol{\Lambda}\right)-\operatorname{tr}\left(\mathbf{B}_{\mathrm{jc}} \boldsymbol{\Delta}\right) \\
& +\sum_{i=1}^{M N} c_{i} \underbrace{\left(\left[\mathbf{A}_{\mathrm{s}}^{T}\right]_{i}^{T} \boldsymbol{\Lambda}\left[\mathbf{A}_{\mathrm{s}}^{H}\right]_{i}+\left[\mathbf{A}_{\mathrm{jc}}^{T}\right]_{i}^{T} \boldsymbol{\Delta}\left[\mathbf{A}_{\mathrm{jc}}^{H}\right]_{i}+\mu_{i}\right)}_{\omega_{i}} \\
& \quad+\mathbf{c}^{T}\left(\operatorname{diag}(\boldsymbol{\mu})+\nu \mathbf{I}+\lambda \mathbf{Q}+\sum_{i=1}^{M} \rho_{i} \mathbf{P}_{t, i}+\sum_{i=1}^{N} \eta_{i} \mathbf{P}_{r, i}\right) \mathbf{c} \\
& \quad-k_{\mathrm{t}} k_{\mathrm{r}} \nu-\lambda k_{\mathrm{r}} k_{\mathrm{t}}\left(k_{\mathrm{r}}+k_{\mathrm{t}}\right) \\
& \quad-N \sum_{i=1}^{M} \rho_{i}+M \sum_{i=1}^{N} \eta_{i} .
\end{aligned}
$$

We minimize $L^{\text {fct }}$ with respect to $\mathbf{c}, \mathbf{X}$, and $\mathbf{Y}$. Noting that $L^{\text {fct }}$ is a mixture of two volume covering ellipsoids in terms of $\mathbf{X}$, and $\mathbf{Y}$ (see p 222 in [29], and Appendix in [30]) and given the set of quadratic forms in $\mathbf{c}$, we arrive at the Lagrange dual function $g^{\text {fct }}$ in 9 .

Theorem 2: Let $f^{\text {jnt }}$ be the optimal value associated with the joint selection 3 and $f^{\mathrm{fct}}$ be that of the factored selection 77. Also, let $k_{\mathrm{r}}^{\mathrm{fct}}, k_{\mathrm{t}}^{\mathrm{fct}}$ be the number of selected transmitters and receivers in the factored selection, and $k^{\text {jnt }}$ the number of jointly selected elements such that $k^{\mathrm{jnt}}=k_{\mathrm{r}}^{\mathrm{fct}} k_{\mathrm{t}}^{\mathrm{fct}}$. Then

$$
f^{\text {jnt }} \geq f^{\mathrm{fct}}
$$




$$
\begin{aligned}
& \left.g^{\mathrm{fct}}(\boldsymbol{\Lambda}, \boldsymbol{\Delta}, \boldsymbol{\mu}, \nu, \lambda, \boldsymbol{\rho}, \boldsymbol{\eta})=\inf _{\mathbf{c}, \mathbf{X}, \mathbf{Y}} L^{\mathrm{fct}}(\mathbf{c}, \mathbf{X}, \mathbf{Y}, \boldsymbol{\Lambda}, \boldsymbol{\Delta}, \boldsymbol{\mu}, \nu, \lambda, \boldsymbol{\rho}, \boldsymbol{\eta})\right\} \\
& \left(\log \operatorname{det}(\boldsymbol{\Lambda})+\log \operatorname{det}(\boldsymbol{\Delta})+2 n+1-\operatorname{tr}\left(\boldsymbol{\Lambda} \mathbf{B}_{\mathrm{s}}\right)-\operatorname{tr}\left(\boldsymbol{\Delta} \mathbf{B}_{\mathrm{jc}}\right)-\frac{1}{4} \boldsymbol{\omega}^{T}\left(\operatorname{diag}(\boldsymbol{\mu})+\nu \mathbf{I}+\lambda \mathbf{Q}+\sum_{i=1}^{M} \rho_{i} \mathbf{P}_{t, i}+\sum_{i=1}^{N} \eta_{i} \mathbf{P}_{r, i}\right)^{-1} \boldsymbol{\omega}\right. \\
& =\left\{\begin{array}{r}
-k_{\mathrm{t}} k_{\mathrm{r}} \nu-\lambda k_{\mathrm{r}} k_{\mathrm{t}}\left(k_{\mathrm{r}}+k_{\mathrm{t}}\right)-k_{\mathrm{r}} \sum_{i=1}^{M} \rho_{i}-k_{\mathrm{t}} \sum_{i=1}^{N} \eta_{i}
\end{array}\right. \\
& (-\infty \quad \text { otherwise. } \\
& \text { if }\left(\operatorname{diag}(\boldsymbol{\mu})+\nu \mathbf{I}+\lambda \mathbf{Q}+\sum_{i=1}^{M} \rho_{i} \mathbf{P}_{t, i}+\sum_{i=1}^{N} \eta_{i} \mathbf{P}_{r, i}\right) \succeq 0, \mathbf{X} \succeq 0, \mathbf{Y} \succeq 0
\end{aligned}
$$

$$
\begin{aligned}
& \left.g^{\mathrm{jnt}}(\boldsymbol{\Lambda}, \boldsymbol{\Delta}, \boldsymbol{\mu}, \nu, \lambda, \boldsymbol{\rho}, \boldsymbol{\eta})=\inf _{\mathbf{c}, \mathbf{X}, \mathbf{Y}} L^{\mathrm{jnt}}(\mathbf{c}, \mathbf{X}, \mathbf{Y}, \boldsymbol{\Lambda}, \boldsymbol{\Delta}, \boldsymbol{\mu}, \nu, \lambda, \boldsymbol{\rho}, \boldsymbol{\eta})\right\} \\
& =\left\{\begin{aligned}
\log \operatorname{det}(\boldsymbol{\Lambda})+\log \operatorname{det}(\boldsymbol{\Delta})+2 n+1-\operatorname{tr}\left(\boldsymbol{\Lambda} \mathbf{B}_{\mathrm{s}}\right)-\operatorname{tr}\left(\boldsymbol{\Delta} \mathbf{B}_{\mathrm{jc}}\right)-\frac{1}{4} \boldsymbol{\omega}^{T}\left(\operatorname{diag}(\boldsymbol{\mu})+\nu \mathbf{I}+\lambda \mathbf{Q}+\sum_{i=1}^{M} \rho_{i} \mathbf{P}_{t, i}+\sum_{i=1}^{N} \eta_{i} \mathbf{P}_{r, i}\right)^{-1} \boldsymbol{\omega} \\
-k^{\mathrm{jnt}} \nu-\lambda k^{\mathrm{jnt}}(M+N)-N \sum_{i=1}^{M} \rho_{i}-M \sum_{i=1}^{N} \eta_{i} \\
\operatorname{if}\left(\operatorname{diag}(\boldsymbol{\mu})+\nu \mathbf{I}+\lambda \mathbf{Q}+\sum_{i=1}^{M} \rho_{i} \mathbf{P}_{t, i}+\sum_{i=1}^{N} \eta_{i} \mathbf{P}_{r, i}\right) \succeq 0, \mathbf{X} \succeq 0, \mathbf{Y} \succeq 0 \\
-\infty \quad \text { otherwise. }
\end{aligned}\right.
\end{aligned}
$$

Proof: Let us recast the joint problem as

$$
\begin{aligned}
\underset{\mathbf{c}}{\max } & f(\mathbf{c}) \\
\text { s.t. } & \\
\mu_{i}: & c_{i}\left(c_{i}-1\right)=0 \quad i=1 \ldots M N, \\
\nu & : \mathbf{c}^{T} \mathbf{c}=k^{\text {jnt }} \\
\lambda & : \mathbf{c}^{T} \mathbf{Q c} \leq k^{\mathrm{jnt}}(M+N) \\
\rho_{i}: & \mathbf{c}^{T} \mathbf{P}_{\mathrm{t}, i} \mathbf{c} \leq N \quad i=1 \ldots M, \\
\eta_{i}: & \mathbf{c}^{T} \mathbf{P}_{\mathrm{r}, i} \mathbf{c} \leq M \quad i=1 \ldots N,
\end{aligned}
$$

We can also reformulate (11) in terms of the equivalent minimization like (8) and derive the Lagrange dual function $g^{\text {jnt }}$ as in $(12)$. By minimization reformulation and employing $\bar{f}^{\text {jnt }}$, and $f^{\text {fct }}$ as the corresponding optimal values, the expression in (10) can be transformed into

$$
\bar{f}^{\mathrm{jnt}} \leq \bar{f}^{\mathrm{fct}} .
$$

Given the upper bounds in 9 and $(12)$, we show 10 by equivalently proving that

$$
g^{\mathrm{jnt}} \leq g^{\mathrm{fct}} .
$$

Now we have that

$$
\mathbf{Q} \succeq 0, \quad \mathbf{P}_{t, i} \succeq 0, i=1, \ldots, M \quad, \quad \mathbf{P}_{r, i} \succeq 0, i=1, \ldots, N
$$

Also, the Karush-Kuhn-Tucker (KKT) conditions [29] imply that

$$
\lambda \geq 0, \quad \boldsymbol{\rho} \geq 0, \quad \boldsymbol{\eta} \geq 0 .
$$

Therefore, by subtracting $(9)$ and $(12)$ we get

$$
\begin{aligned}
& g^{\mathrm{fct}}-g^{\mathrm{jnt}}= \lambda\left(k^{\mathrm{jnt}}\left((M+N)-\left(k_{\mathrm{r}}^{\mathrm{fct}}+k_{\mathrm{t}}^{\mathrm{fct}}\right)\right)\right) \\
&+\sum_{i=1}^{M} \rho_{i}\left(N-k_{\mathrm{r}}^{\mathrm{fct}}\right)+\sum_{i=1}^{N} \eta_{i}\left(M-k_{\mathrm{t}}^{\mathrm{fct}}\right) \\
& \geq 0
\end{aligned}
$$

The factored selection operates on a subset of solutions that is included in the joint selection, and hence may not achieve the same optimal solution that is guaranteed by joint selection. Nonetheless, selecting a subset of transmitters allows the available total transmit power to be allocated only to the chosen elements. This is in contrast to the joint selection problem where all transmitters must be operational to guarantee that all matched filters are available for selection. Thus, assuming a total available transmit power $P_{e}=P_{T}$, the transmit power per element in the factored case is $P_{e}=\frac{P_{t}}{k_{\mathrm{t}}}$ as opposed to $\frac{P_{t}}{M}$ for the joint selection case. It is important to note, however that increasing the allocation of transmit power per element may be restricted by the hardware limitations of the components in the RF chain, such as amplifier linear range. This may limit the gain achievable by the factored approach.

\section{Matched Filter Constrained Selection}

We can adjust the transmitter power and SNR by a factored selection. Moreover, the number of receivers is decreased, which leads to a considerable hardware reduction. Since the number of transmitters is reduced in a factored selection, the spatial diversity is reduced significantly [31]. To preserve the spatial diversity provided by MIMO arrays but still reduce hardware and computation overheads, we propose restricting 
the number of matched filters in each receiver, as well as the number of receivers, in a matched filter constrained (MFC) selection strategy [32]. Using this, we decrease the number of $\mathrm{RF}$ front-ends on the receive end (stage 2 in Fig 1 ) as well as the required processing blocks in DSP (stage 3 in Fig 1 ).

We specify the MFC selection to select $k_{\mathrm{m}}$ matched filters in $k_{\mathrm{r}}$ receivers as follows

$$
\begin{aligned}
\underset{\mathbf{c}}{\max } & f(c) \\
\text { s.t. } & c_{i}^{2}-c_{i}=0 \quad i=1 \ldots M N, \\
& \mathbf{c}^{T} \mathbf{c}=k_{\mathrm{m}} k_{\mathrm{r}}, \\
& \mathbf{c}^{T} \mathbf{Q}_{\mathrm{r}} \mathbf{c}=k_{\mathrm{m}}^{2} k_{\mathrm{r}}, \\
& \mathbf{c}^{T} \mathbf{P}_{\mathrm{t}, i} \mathbf{c}<=k_{\mathrm{r}} \quad i=1 \ldots M \\
& \mathbf{c}^{T} \mathbf{P}_{\mathrm{r}, i} \mathbf{c}<=k_{\mathrm{m}} \quad i=1 \ldots N
\end{aligned}
$$

where $\mathbf{Q}_{\mathrm{r}}$ is

$$
\mathbf{Q}_{\mathrm{r}}=\mathbf{P}_{\mathrm{r}} \mathbf{P}_{\mathrm{r}}^{T}, \quad \mathbf{P}_{\mathrm{r}}=\left[\mathbf{v}_{\mathrm{r}, 1}, \ldots, \mathbf{v}_{\mathrm{r}, N}\right]
$$

Comparing (13) with (7) makes it obvious that the factored selection is a special case of the MFC selection. Therefore, for concision we state the following theorem without proof.

Theorem 3: Let $f^{\mathrm{mfc}}$ be the optimal value of the MFC selection 13 and $f^{\text {jnt }}$ the optimal value of the joint selection (11), and $f^{\text {fct }}$ the optimal value of the factored selection (7). Moreover, let $k_{\mathrm{m}}^{\mathrm{mfc}}$ be the number of selected matched-filters and receivers in MFC selection, $k_{\mathrm{r}}^{\mathrm{mfc}}$, let $k_{\mathrm{r}}^{\mathrm{fct}}, k_{\mathrm{t}}^{\mathrm{fct}}$ be the number of selected transmitters and receivers respectively, in factored selection, and $k^{\mathrm{jnt}}$ be the number of jointly selected elements such that $k^{\mathrm{jnt}}=k_{\mathrm{m}}^{\mathrm{mfc}} k_{\mathrm{r}}^{\mathrm{mfc}}=k_{\mathrm{r}}^{\mathrm{fct}} k_{\mathrm{t}}^{\mathrm{fct}}$. Then

$$
f^{\mathrm{jnt}} \geq f^{\mathrm{mfc}} \geq f^{\mathrm{fct}}
$$

\section{Hybrid Selection}

By the giving up of spatial diversity described for factored selection, there is a benefit for transmit power and selecting the optimum subset of matched filters with a decrease in the number of transmitters and receivers. On the other hand, in MFC selection we maintain spatial diversity at the expense of transmit power. In this subsection, we propose integrating these two methods into a hybrid algorithm by which the MIMO array is entirely controlled. In a MIMO radar comprising $M$ transmitters and $N$ receivers, the hybrid selection finds the optimum subset including $k=k_{\mathrm{r}} k_{\mathrm{m}}$ matched filters such that exactly $k_{\mathrm{m}}$ out of $k_{\mathrm{t}}$ active transmitters are used in each $k_{\mathrm{r}}$ receivers. By adopting the same methodology introduced in (6), we define the hybrid selection as

$$
\begin{aligned}
\underset{\mathbf{c}}{\max } & f(\mathbf{c}) \\
\text { s.t. } & c_{i}^{2}-c_{i}=0 \quad i=1 \ldots M N, \\
& \mathbf{c}^{T} \mathbf{c}=k_{\mathrm{m}} k_{\mathrm{r}}, \\
& \mathbf{c}^{T} \mathbf{P}_{\mathrm{t}, i} \mathbf{c} \in\left\{0, k_{\mathrm{r}}\right\} \quad i=1 \ldots M \\
& \operatorname{card}\left(\mathbf{c}^{T} \mathbf{P}_{r}\right) \leq k_{\mathrm{t}}
\end{aligned}
$$

where card $(\mathbf{x})$ denotes the cardinality, the number of non-zero elements of vector $\mathbf{x}$. We first relax (14c) via $(15 \mathrm{~d})$, and $(15 \mathrm{f})$ in the following optimization

$$
\begin{array}{cl}
\max _{\mathbf{c}} & f(\mathbf{c}) \\
\text { s.t. } & c_{i}^{2}-c_{i}=0 \quad i=1 \ldots M N, \\
& \mathbf{c}^{T} \mathbf{c}=k_{\mathrm{m}} k_{\mathrm{r}} \\
& \operatorname{card}\left(\mathbf{c}^{T} \mathbf{P}_{r}\right) \leq k_{\mathrm{t}} \\
& \mathbf{c}^{T} \mathbf{Q}_{r} \mathbf{c}=k_{\mathrm{m}}^{2} k_{\mathrm{r}} \\
& \mathbf{c}^{T} \mathbf{P}_{\mathrm{t}, i} \mathbf{c} \leq k_{\mathrm{r}} \quad i=1 \ldots M \\
& \mathbf{c}^{T} \mathbf{P}_{\mathrm{r}, i} \mathbf{c} \leq k_{\mathrm{m}} \quad i=1 \ldots N
\end{array}
$$

where $\mathbf{P}_{\mathrm{t}}$ is

$$
\mathbf{P}_{\mathrm{t}}=\left[\mathbf{v}_{\mathrm{t}, 1}, \ldots, \mathbf{v}_{\mathrm{t}, M}\right]
$$

The cardinality constraint $[15 \mathrm{c})$, is a nonconvex constraint. The best relaxation strategy for a cardinality or norm- 0 constraint is a norm-1 constraint [33], which is already met by (15b]. To tackle this constraint, we employ alternative constraints described in the following theorem.

Theorem 4: Let $\mathbf{c}$ be the selection vector associated with the hybrid selection satisfying $15 \mathrm{a}$ to $15 \mathrm{~b}$. Then, we can replace $15 \mathrm{c}$ by the following inequality constraints

$$
\frac{\left(k_{\mathrm{r}} k_{\mathrm{m}}\right)^{2}}{k_{\mathrm{t}}} \leq \mathbf{c}^{T} \mathbf{Q}_{\mathrm{t}} \mathbf{c} \leq k_{\mathrm{r}}^{2} k_{\mathrm{m}},
$$

where $\mathbf{Q}_{\mathrm{t}}=\mathbf{P}_{\mathrm{t}} \mathbf{P}_{\mathrm{t}}^{T}$.

Proof: See Appendix B

We relax 15c) through (16c), and ultimately reformulate the hybrid selection as follows

$$
\begin{array}{cl}
\max _{\mathbf{c}} & f(\mathbf{c}) \\
\text { s.t. } & c_{i}^{2}-c_{i}=0 \quad i=1 \ldots M N, \\
& \mathbf{c}^{T} \mathbf{c}=k_{\mathrm{m}} k_{\mathrm{r}} \\
& \frac{\left(k_{\mathrm{r}} k_{\mathrm{m}}\right)^{2}}{k_{\mathrm{t}}} \leq \mathbf{c}^{T} \mathbf{Q}_{\mathrm{t}} \mathbf{c} \leq k_{\mathrm{r}}^{2} k_{\mathrm{m}}, \\
& \mathbf{c}^{T} \mathbf{Q}_{\mathrm{r}} \mathbf{c}=k_{\mathrm{m}}^{2} k_{\mathrm{r}} \\
& \mathbf{c}^{T} \mathbf{P}_{\mathrm{r}, i} \mathbf{c} \leq k_{\mathrm{m}} \quad i=1 \ldots N \\
& \mathbf{c}^{T} \mathbf{P}_{\mathrm{t}, i} \mathbf{c} \leq k_{\mathrm{r}} \quad i=1 \ldots M
\end{array}
$$

In the following theorem, we study the performance of the hybrid selection with respect to the rest of the modes.

Theorem 5: Let $f^{\text {fct }}$ be the optimal value of the factored selection (7), $f^{\text {hyb }}$ the MFC selection (13), and $f^{\text {hyb }}$ the optimal value of the hybrid selection 116 . Also, let $k_{\mathrm{r}}^{\mathrm{fct}}, k_{\mathrm{t}}^{\mathrm{fct}}$ be the number of selected transmitters and receivers in factored selection, $k_{\mathrm{m}}^{\mathrm{mfc}}, k_{\mathrm{r}}^{\mathrm{mfc}}$ the number of selected matched filters and receivers in MFC selection, and $k_{\mathrm{m}}^{\mathrm{hyb}}, k_{\mathrm{r}}^{\mathrm{hyb}}, k_{\mathrm{t}}^{\mathrm{hyb}}$ the number of selected matched filters, receivers, and the number of allowed transmitters in hybrid selection. Moreover, suppose 
that $k$ elements are selected in each selection strategy subject to the following conditions

$$
\begin{aligned}
& A: k=k_{\mathrm{r}}^{\mathrm{fct}} k_{\mathrm{t}}^{\mathrm{fct}}=k_{\mathrm{m}}^{\mathrm{mfc}} k_{\mathrm{r}}^{\mathrm{mfc}}=k_{\mathrm{m}}^{\mathrm{hyb}} k_{\mathrm{r}}^{\mathrm{hyb}} \\
& B: k_{\mathrm{r}}^{\mathrm{fct}}=k_{\mathrm{r}}^{\mathrm{mfc}}=k_{\mathrm{r}}^{\mathrm{hyb}} \\
& C: k_{\mathrm{t}}^{\mathrm{fct}}=k_{\mathrm{m}}^{\mathrm{mfc}}=k_{\mathrm{m}}^{\mathrm{hyb}} \\
& D: k_{\mathrm{m}}^{\mathrm{hyb}} \leq k_{\mathrm{t}}^{\mathrm{hyb}}
\end{aligned}
$$

Then,

$$
f^{\text {fct }} \leq f^{\text {hyb }} \leq f^{\mathrm{mfc}}
$$

Proof: We can rewrite (7) as

$$
\begin{array}{rl}
\underset{\mathbf{c}}{\max _{1}} & f(\mathbf{c}) \\
\text { s.t. } & \\
\mu_{i}: & c_{i}^{2}-c_{i}=0 \quad i=1 \ldots M N, \\
\nu: & \mathbf{c}^{T} \mathbf{c}=k_{\mathrm{t}} k_{\mathrm{r}} \\
\lambda: & \mathbf{c}^{T} \mathbf{Q}_{\mathrm{r}} \mathbf{c}=k_{\mathrm{t}}^{2} k_{\mathrm{r}} \\
\kappa & : \quad \mathbf{c}^{T} \mathbf{Q}_{\mathrm{t}} \mathbf{c}=k_{\mathrm{t}} k_{\mathrm{r}}^{2} \\
\rho_{i}: & \mathbf{c}^{T} \mathbf{P}_{\mathrm{t}, i} \mathbf{c} \leq k_{\mathrm{r}} \quad i=1 \ldots M \\
\eta_{i}: & \mathbf{c}^{T} \mathbf{P}_{\mathrm{r}, i} \mathbf{c} \leq k_{\mathrm{t}} \quad i=1 \ldots N
\end{array}
$$

while, we revise (14) as:

$$
\begin{aligned}
\underset{\mathbf{c}}{\max } & f(\mathbf{c}) \\
\text { s.t. } & \\
\mu_{i}: & c_{i}^{2}-c_{i}=0 \quad i=1 \ldots M N, \\
\nu: & \mathbf{c}^{T} \mathbf{c}=k_{\mathrm{t}} k_{\mathrm{r}} \\
\lambda: & \mathbf{c}^{T} \mathbf{Q}_{\mathrm{r}} \mathbf{c}=k_{\mathrm{t}}^{2} k_{\mathrm{r}} \\
\kappa: & \mathbf{c}^{T} \mathbf{Q}_{\mathrm{t}} \mathbf{c}=k_{\mathrm{t}} k_{\mathrm{r}}^{2} \\
\sigma: & \mathbf{c}^{T} \mathbf{Q}_{\mathrm{t}} \mathbf{c}<k_{\mathrm{r}}^{2} k_{\mathrm{t}} \\
\tau & : \quad \mathbf{c}^{T} \mathbf{Q}_{\mathrm{t}} \mathbf{c} \geq \frac{\left(k_{\mathrm{r}} k_{\mathrm{m}}\right)^{2}}{k_{\mathrm{t}}} \\
\rho_{i}: & \mathbf{c}^{T} \mathbf{P}_{\mathrm{t}, i} \mathbf{c} \leq k_{\mathrm{r}} \quad i=1 \ldots M \\
\eta_{i}: & \mathbf{c}^{T} \mathbf{P}_{\mathrm{r}, i} \mathbf{c} \leq k_{\mathrm{m}} \quad i=1 \ldots N .
\end{aligned}
$$

By computing the Lagrangian dual function of (17a), and (18) we get

$$
g^{\mathrm{hyb}}-g^{\mathrm{fct}}=-\frac{1}{4} \boldsymbol{\omega}^{T}\left((\sigma+\tau) \mathbf{Q}_{\mathrm{t}}\right) \boldsymbol{\omega}-\sigma k_{\mathrm{r}}^{2} k_{\mathrm{t}}-\tau \frac{\left(k_{\mathrm{r}} k_{\mathrm{m}}\right)^{2}}{k_{\mathrm{t}}} .
$$

Due to the KKT conditions, we note that

$$
\sigma \geq 0, \quad \tau \geq 0
$$

Therefore,

$$
g^{\mathrm{hyb}}-g^{\mathrm{fct}} \leq 0
$$

and consequently

$$
f^{\mathrm{fct}} \leq f^{\mathrm{hyb}} .
$$

Following the same process for comparing $f^{\text {hyb }}$ and $f^{\mathrm{mfc}}$ yields

$$
f^{\text {hyb }} \leq f^{\mathrm{mfc}}
$$

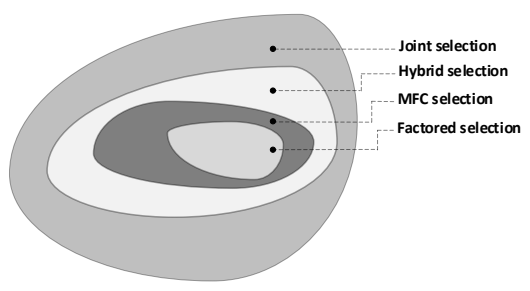

Fig. 2. Geometric illustration of feasible regions and relationship of different selection methods.

\section{E. Performance and Complexity Analysis}

By Theorem 3, and Theorem 5, we conclude the following, in terms of the output SINR,

$$
f^{\mathrm{fct}} \leq f^{\mathrm{hyb}} \leq f^{\mathrm{mfc}} \leq f^{\mathrm{jnt}}
$$

Also, based on this conclusion the feasible regions for different selection modes given by intersection of positive semidefinite cones can be illustrated as in Fig. 2

The computational complexity of the minimum volume ellipsoid problem when solving via barrier-generated pathfollowing interior-point method with $n$ variables and $m$ constraints can be approximated by $O\left(m^{2.5}\left(n^{2}+m\right)\right)$ (see Theorem 6.5.1 in [34]). We can represent the joint selection with at least $m^{\text {jnt }}=2$ constraints. However, the factored and MFC selections need at least $m^{\text {fct }}=m^{\text {mfc }}=3+M+N$ constraints, and the hybrid selection requires $m^{\mathrm{fct}}=m^{\mathrm{mfc}}=5+M+N$ constraints. Hence, the computational complexities can be summarized as

$$
O^{\mathrm{jnt}} \leq O^{\mathrm{fct}}=O^{\mathrm{mfc}} \leq O^{\mathrm{hyb}}
$$

\section{RELAXATION AND APPROXIMATION}

In the previous section, we expressed the selection methods as a difference log-determinant maximization with quadratic constraints. Although we decoupled the factored problem in subsection III-B, and relaxed the norm constraints in subsection III-D, the problem still is not tractable due to the nonconvexity of (a) a difference of concave functions, (b) quadratic equalities, e.g. (7a)- (7c), and (c) the quadratic inequality from constructing a nonconvex set, e.g. (18g). To handle these nonconvexities, we propose a variation of sequential convex programming (SCP) with an exact penalty approach [35]. Let the primal problem be defined as

$$
\begin{array}{rl}
\max _{\mathbf{c}} & f(\mathbf{c}) \\
\text { s.t. } & c_{i} \in\{0,1\} \quad i=1 \ldots M N, \\
& g_{1}(\mathbf{c}) \geq 0 \\
& g_{i}(\mathbf{c}) \leq 0, \quad i=1, \ldots, \ell, \\
& e_{i}(\mathbf{c})=0, \quad i=1, \ldots, \jmath .
\end{array}
$$

Where $f(\mathbf{c})$ is a concave-concave function, $g(\mathbf{c})$, and $e(\mathbf{c})$ represent quadratic functions. By relaxing the binary constraint 
and using a convex local approximation we specify the problem in the $\ell$-th iteration as

$$
\begin{aligned}
\max _{\mathbf{c}^{(l)}} & \hat{f}\left(\mathbf{c}^{(l)}\right)+\psi \sum_{i=1}^{\jmath} e_{i}\left(\mathbf{c}^{(l)}\right) \\
\text { s.t. } & 0 \leq c_{i}^{(i)} \leq 1 \quad i=1 \ldots M N, \\
& \hat{g}_{1}\left(\mathbf{c}^{(l)}\right) \geq 0 \\
& g_{i}\left(\mathbf{c}^{(l)}\right) \leq 0, \quad i=1, \ldots, \ell .
\end{aligned}
$$

Where we use a first Taylor expansion to get an affine approximation over the trust region of a box around the current point. Employing this for $f(\mathbf{c})$ we will have

$$
\begin{aligned}
\hat{f}\left(\mathbf{c}^{(l)}\right) & =f_{1}\left(\mathbf{c}^{(l)}\right)-f_{2}\left(\mathbf{c}^{(l)}\right)-\nabla f_{2}\left(\mathbf{c}^{(l)}\right)\left(\mathbf{c}^{(l)}-\mathbf{c}^{(l-1)}\right) \\
& =\log \operatorname{det}\left(\mathbf{A}_{\mathrm{s}}^{H} \operatorname{diag}\left(\mathbf{c}^{(l)}\right) \mathbf{A}_{\mathrm{s}}+\mathbf{B}_{\mathrm{s}}\right) \\
& -\log \operatorname{det}\left(\mathbf{A}_{\mathrm{jc}}^{H} \operatorname{diag}\left(\mathbf{c}^{(l-1)}\right) \mathbf{A}_{\mathrm{jc}}+\mathbf{B}_{\mathrm{jc}}\right) \\
& -\operatorname{diag}\left(\mathbf{a}_{s}^{H}\left(\mathbf{A}_{\mathrm{jc}}^{H} \operatorname{diag}\left(\mathbf{c}^{(l-1)}\right) \mathbf{A}_{\mathrm{jc}}+\mathbf{B}_{\mathrm{jc}}\right)^{-1} \mathbf{a}_{s}\right) .
\end{aligned}
$$

We also use an affine approximation for $g_{1}(\mathbf{c})$ to tackle the nonconvex constraint

$$
\begin{aligned}
& \hat{g}_{1}\left(\mathbf{c}^{(k)}\right)=g_{1}\left(\mathbf{c}^{(k)}\right)+\nabla g_{1}\left(\mathbf{c}^{(k)}\right)\left(\mathbf{c}^{(k)}-\mathbf{c}^{(k-1)}\right. \\
& =\mathbf{c}^{(k)^{T}} \mathbf{W}_{1} \mathbf{c}^{(k)}+r_{1}+\left(\left(\mathbf{W}_{1}+\mathbf{W}_{1}^{T}\right) \mathbf{c}^{(k)}\right)\left(\mathbf{c}^{(k)}-\mathbf{c}^{(k-1)}\right)
\end{aligned}
$$

After solving the relaxed version of the problem by an SCP procedure, we find the suboptimal solution of the original problem by an appropriate randomized rounding strategy.

Let $\left(\mathbf{c}^{\star}\right)$ be the optimal value obtained by relaxation and SCP. Also, assume $\mathbf{z} \in \mathbf{R}^{M N}$ is a Gaussian variable with distribution $\mathbf{z} \sim \mathcal{N}(\mu, \Sigma)$. Then, the following maximization

$$
\begin{array}{rl}
\underset{\mathbf{c}}{\max _{\mathbf{c}}} & \mathbb{E}(f(\mathbf{c})) \\
\text { s.t. } & \mathbb{E}\left(c_{i}\right) \in\{0,1\} \quad i=1 \ldots M N, \\
& \mathbb{E}\left(g_{1}(\mathbf{c})\right) \geq 0 \\
& \mathbb{E}\left(g_{i}(\mathbf{c})\right) \leq 0, \quad i=1, \ldots, \ell, \\
& \left.\mathbb{E}\left(h_{i}(\mathbf{c})\right)=0, \quad i=1, \ldots,\right\},
\end{array}
$$

is solved by $\mathbf{z}$ for $\mu=\mathbf{c}^{\star}$. As for $\Sigma$, we use $\Sigma=$ $\operatorname{diag}\left(\left[\operatorname{var}\left(\mathbf{c}_{i}\right)\right]\right)$ where $\mathbf{c}_{i}$ is a vector comprising the sequence of solutions of (19). We take a sample from $\mathbf{z}$ for a sufficent number of times and and keep the best sample yielding the maximum value. Nevertheless, this direct sampling does not immediately provide a feasible point regarding the embedded quadratic constraints. Hence, we need to project the direct sampled vector onto the constraints feasible set. To do this we add one more step after sampling, where we find the projected point $\hat{\mathbf{z}}$ via the following optimization

$$
\begin{array}{ll}
\min _{\hat{\mathbf{z}}} & \|\hat{\mathbf{z}}-\mathbf{z}\| \\
\text { s.t. } & c_{i} \in\{0,1\} \quad i=1 \ldots M N, \\
& g_{1}(\mathbf{c}) \geq 0 \\
& g_{i}(\mathbf{c}) \leq 0, \quad i=1, \ldots, \ell, \\
& h_{i}(\mathbf{c})=0, \quad i=1, \ldots, j .
\end{array}
$$

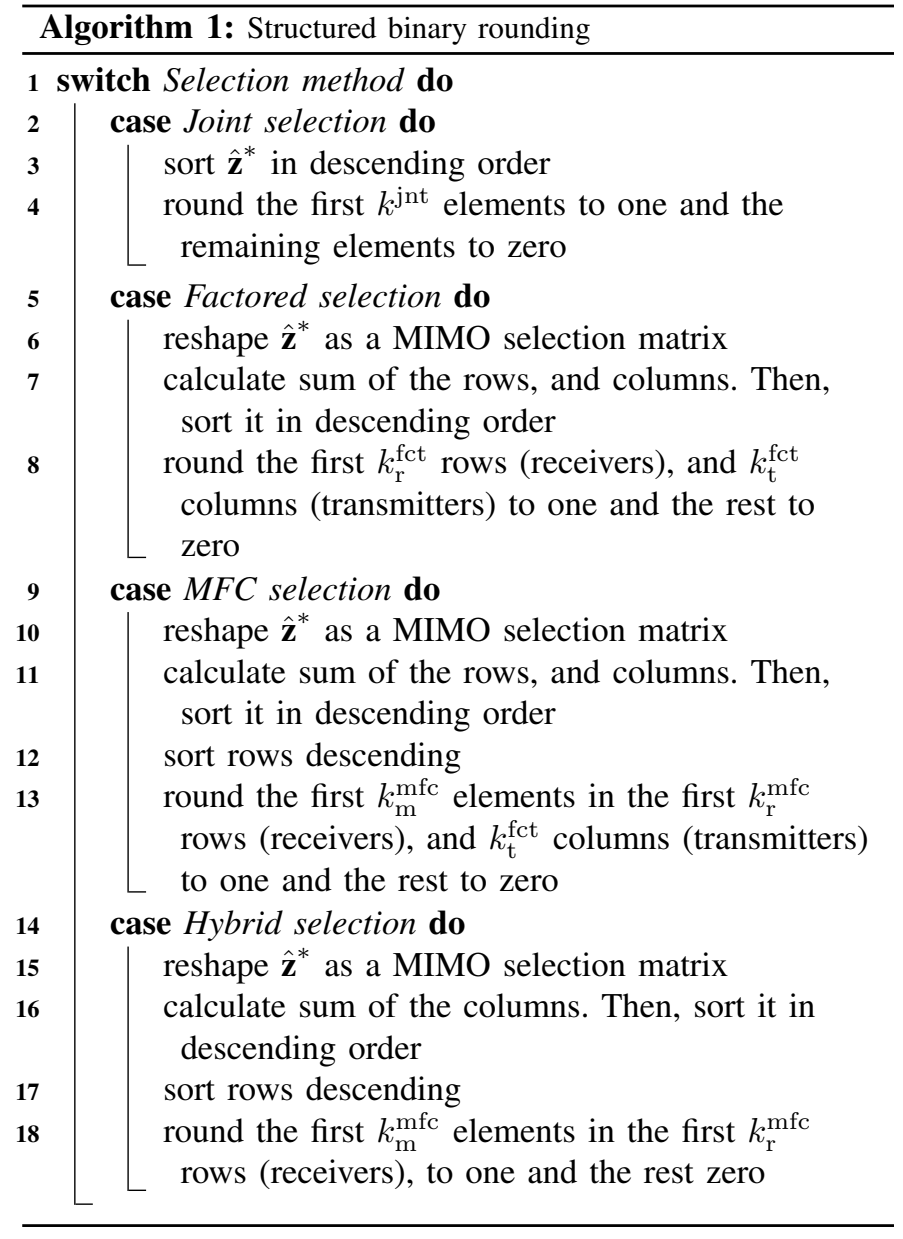

This optimization is another form of binary programming. Heuristically, we approximate this problem in two successive steps. We first find the projected sample by applying a relaxation as follows

$$
\begin{array}{ll}
\min _{\hat{\mathbf{z}}} & \|\hat{\mathbf{z}}-\mathbf{z}\| \\
\text { s.t. } & 0 \leq c_{i} \leq 1 \quad i=1 \ldots M N, \\
& g_{i}(\mathbf{c}) \leq 0, \quad i=1, \ldots, \ell, \\
& h_{i}(\mathbf{c}) \leq 0, \quad i=1, \ldots, \jmath .
\end{array}
$$

Then the best feasible projected sample $\hat{\mathbf{z}}^{*}$ is obtained through successive evaluations of the cost function. We then employ a structured rounding strategy to round $\hat{\mathbf{z}}^{*}$ to the nearest binary point, while still meeting the structure of the selection vector for different selection methods. The corresponding structured rounding for each selection method is listed in Algorithm 1 while the final algorithm is summarized in Algorithm 2.

\section{Simulation}

To evaluate the performance of the proposed selection methods, we use a uniform linear collocated MIMO phased array comprising 5 transmitters $(M=5)$, and 5 receivers $(N=5)$. We use the inter-element spacing of $d_{\mathrm{r}}=\frac{\lambda}{2}$ for the elements in the receive array. To maintain a non-overlapping virtual array and maximum aperture we place the transmit antennas $d_{\mathrm{t}}=N d_{\mathrm{r}}$ apart. We include two jamming signals 
with azimuth angles of $\theta_{j}=[20,50]$ degrees and powers of $13 \mathrm{dBW}$. We vary the azimuth angle of the received signal, $\theta_{s}$ from $0^{\circ}$ to $90^{\circ}$, while the power is fixed at $20 \mathrm{dBW}$. Also, we assume that clutter is contributed from angles between $0^{\circ}$ and $90^{\circ}$, and we use a low rank model with rank 5 and a total clutter-to-noise ratio of $13 \mathrm{~dB}$. We define the problem such that 12 elements are selected in total according to different selection methods, such that for joint selection $k^{\mathrm{jnt}}=12$, and for the factored selection $k_{\mathrm{t}}^{\mathrm{fct}}=3, k_{\mathrm{r}}^{\mathrm{fct}}=4$. The selection criteria for MFC and hybrid selections are $k_{\mathrm{m}}^{\mathrm{mfc}}=3, k_{\mathrm{r}}^{\mathrm{mfc}}=4$ and $k_{\mathrm{t}}^{\text {hyb }}=4, k_{\mathrm{m}}^{\text {hyb }}=3, k_{\mathrm{r}}^{\text {hyb }}=4$. The maximum $\mathrm{SINR}_{\text {out }}$ is first calculated by an exhaustive search for each of the strategies and the suboptimal solution is then obtained from Algorithm 2 For each direction, we used the SDPT3 solver embedded in CVX [36] to solve (19) for 10 iterations, and then proceeded to take 1000 samples from a random distribution as described in Algorithm 2. The achieved values for SINR out resulting from the exhaustive search and proposed method are depicted in Figs. 3(a) 3(d) along with the corresponding SINR $_{\text {out }}$ for the full array achieved by an MVDR beamformer. The approximated value is close to the exhaustive search, confirming that a good approximation ratio is achieved by the proposed relaxation strategy. For the factored and hybrid selection two sets of curves are shown in Figs. 3(b) 3(d) to demonstrate the effect of the power adjustment. The additional transmit power afforded by allocating the total available power to fewer transmit waveforms improves the SINR out $_{\text {signifi- }}$ cantly in these modes of selection.

Next, we show in Fig. 4 the selected elements yielded by each of the selection strategies. In this example, we fix the impinging signal direction at $\theta_{s}=18^{\circ}$. We can see from Fig. 4 (a) that all of the transmitters and receivers must be operational in the joint selection in order to be able to select from the corresponding matched-filters. On the other hand, exactly two transmitters and two receivers were deactivated in factored selection as shown in Fig. 4(b). For MFC selection three matched filters are selected precisely at four receivers. While in hybrid selection the three matched filters are selected only from 4 possible options as Tx-1 is deactivated.

We now compare the performances of the selection methods. In Fig. 5 we plot the SINR $_{\text {out }}$ achieved by the different selection methods via exhaustive search, While in Fig. 6 the optimal values are shown for the proposed optimization. Ignoring the power adjustment option, we see in both figures that joint selection achieves the highest SINR $_{\text {out }}$ followed by MFC, hybrid, and then factored selection, which verifies

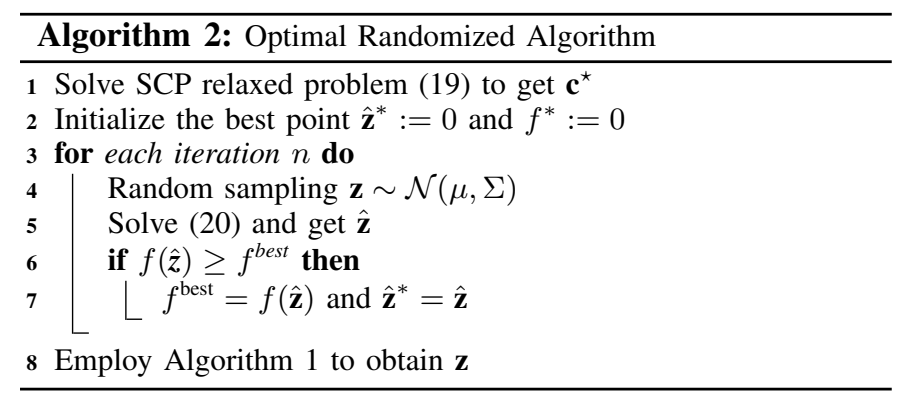

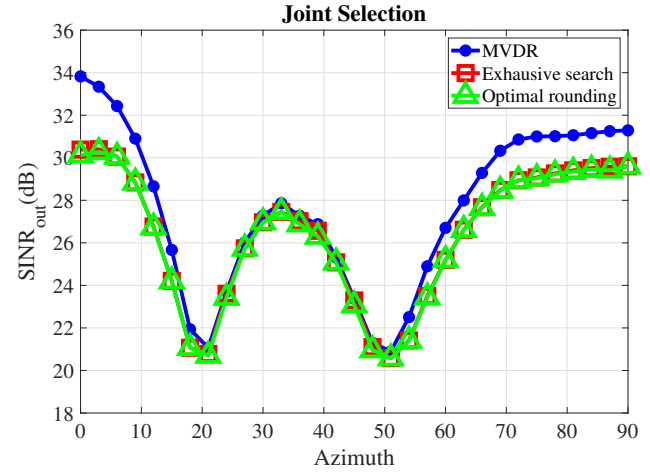

(a)

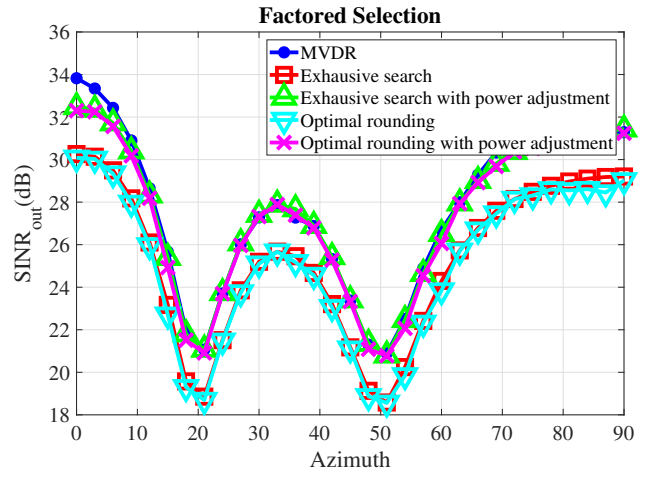

(b)

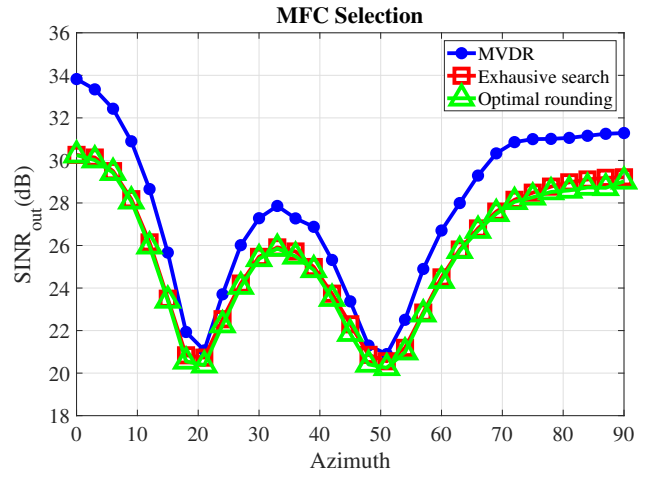

(c)

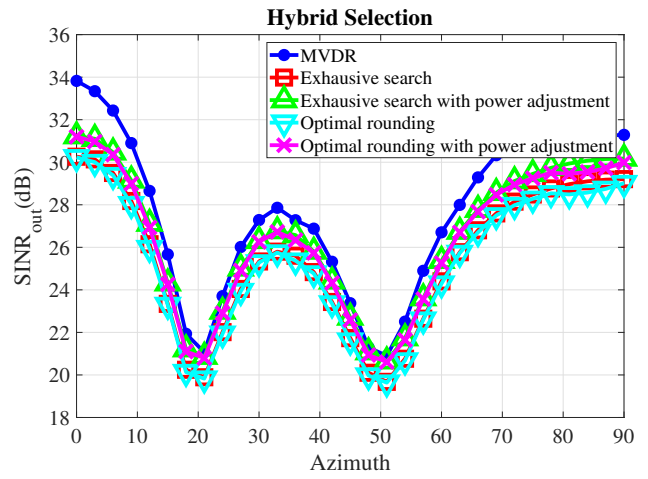

(d)

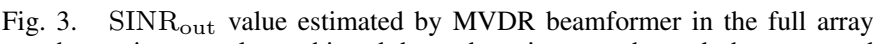
vs. the optimum value achieved by exhaustive search, and the proposed optimization for (a) Joint selection, (b) Factored selection, (c) MFC selection, and (d) Hybrid selection. 


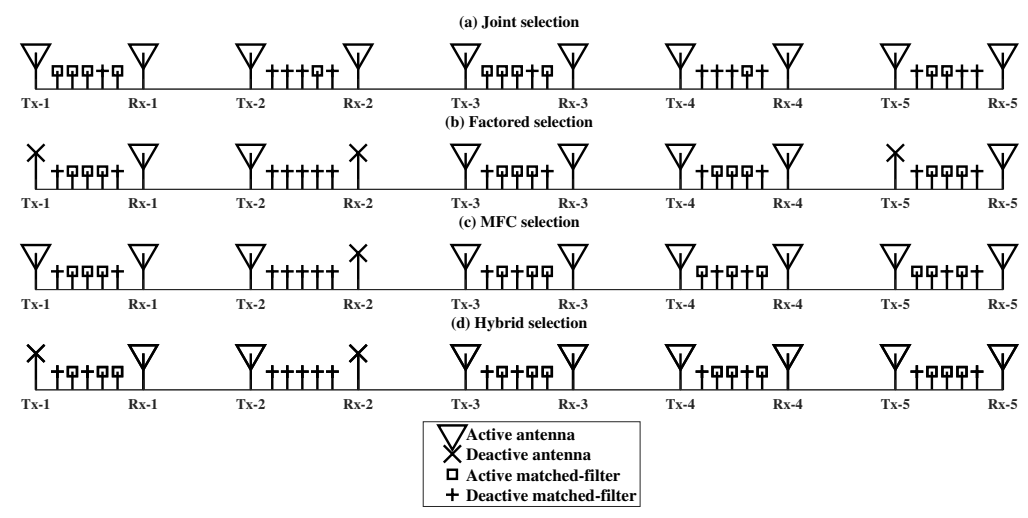

Fig. 4. Illustration of the output selection in different selection modes.

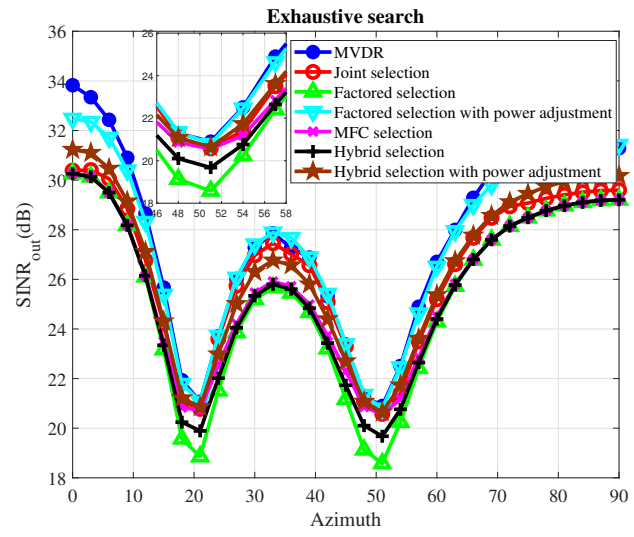

Fig. 5. SINR out value estimated by MVDR beamformer in the full array vs. the optimum value achieved by exhaustive search in different selection modes.

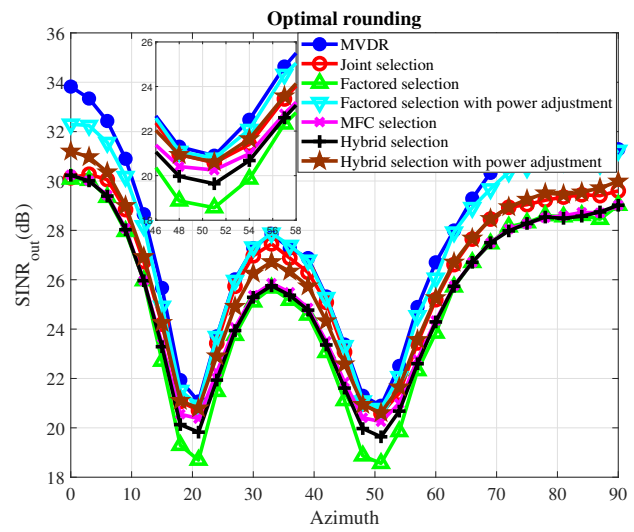

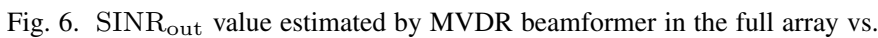
the optimum value achieved by proposed optimization in different selection modes.

Theorems (2) and (5). However, when the power adjustment for factored and hybrid selections is included, the increased transmit signal power means that the factored and hybrid selections surpass the joint mode.

The above example employed a $5 \times 5$ array to permit us to compare the performance to the exhaustive search. Now we demonstrate the performance with a larger array such that the exhaustive search is not possible. To this end, we employ a $10 \times 10$ array. Additionally, we increase the number of jammers to 5 and the clutter rank to 10 . The five jamming signals have azimuth angles $\theta_{j}=[0,5,15,25,30]$ degrees and powers of $13 \mathrm{dBW}$, and the total clutter-to-noise ratio is set to $13 \mathrm{~dB}$. The SOI is assumed to have a power of $20 \mathrm{dBW}$ at the reciever and its azimuth is varied between $0^{\circ}$ to $30^{\circ}$. We select 54 elements in total such that for joint selection $k^{\mathrm{jnt}}=54$, and for the factored selection $k_{\mathrm{t}}^{\mathrm{fct}}=6, k_{\mathrm{r}}^{\mathrm{fct}}=9$. The selection criteria for MFC are $k_{\mathrm{m}}^{\mathrm{mfc}}=6, k_{\mathrm{r}}^{\mathrm{mfc}}=9$ and for the hybrid strategy $k_{\mathrm{t}}^{\text {hyb }}=7, k_{\mathrm{m}}^{\text {hyb }}=6, k_{\mathrm{r}}^{\mathrm{hyb}}=9$. The optimal values achieved by the proposed optimization are depicted in Fig. 7 Firstly, observe the excellent performance achieved for a larger array with a denser interference environment. The proposed selection strategies exhibit similar trends to the previous, smaller example, with the joint as well as factored and hybrid strategies (with power adjustment) being comparable to the MVDR with almost half the number of elements.

In the following example, we again use the $5 \times 5$ array of the first example. We fix the signal direction $\theta_{s}=18^{\circ}$ and solve the selection problem for an increasing subset of antennas ranging from 2 to 25 . The preset values for all the selection methods are listed in Table [1] As is revealed in Fig. 8, the joint selection outperforms the rest of selection methods when power adjustment is not employed, followed by MFC, hybrid and factored approaches. When the transmit power is adjusted, we see that the factored and hybrid approaches are

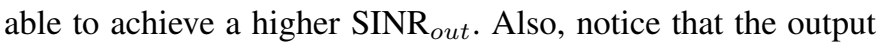
SINR given by the selection remains comparable with that of the full array even when a significantly smaller number of pairs are used. For instance selecting 15 out of 25 elements would substantially reduce the dimensionality and hardware cost but would result in a SINR out loss with respect to the full array of less than $0.5 \mathrm{~dB}$ for joint selection and 2.24 $\mathrm{dB}$ for factored selection (when the power is not adjusted). Adjusting the transmit power, however, can have a significant effect giving, even showing an improvement over the full array.

\section{CONCLUSION}

In this paper, we formulated the antenna selection in MIMO arrays with beamforming to mitigate interference signals in the form of multiple jamming signals and clutter. We devised four different selection approaches to control different aspects of a MIMO system. We cast the problem as a determinant 
TABLE I

THE NUMBER OF SELECTED ELEMENTS IN EACH SELECTION METHOD FOR THE RESULTS SHOWN IN FIG. 8

\begin{tabular}{|c|c|c|c|c|c|c|c|}
\hline$k_{\mathrm{jnt}}$ & $k_{\mathrm{t}}^{\text {fct }}$ & $k_{\mathrm{r}}^{\text {fct }}$ & $k_{\mathrm{m}}^{\mathrm{mfc}}$ & $k_{\mathrm{r}}^{\mathrm{mfc}}$ & $k_{\mathrm{t}}^{\text {hyb }}$ & $k_{\mathrm{r}}^{\text {hyb }}$ & $k_{\mathrm{m}}^{\text {hyb }}$ \\
\hline 2 & 1 & 2 & 1 & 2 & 4 & 2 & 1 \\
\hline 3 & 1 & 3 & 1 & 3 & 4 & 3 & 1 \\
\hline 4 & 2 & 2 & 2 & 2 & 4 & 2 & 2 \\
\hline 5 & 1 & 5 & 1 & 5 & 4 & 5 & 1 \\
\hline 6 & 2 & 3 & 2 & 3 & 4 & 3 & 2 \\
\hline 8 & 2 & 4 & 2 & 4 & 4 & 4 & 2 \\
\hline 9 & 3 & 3 & 3 & 3 & 4 & 3 & 3 \\
\hline 10 & 2 & 5 & 2 & 5 & 4 & 5 & 2 \\
\hline 12 & 3 & 4 & 3 & 4 & 4 & 4 & 3 \\
\hline 15 & 3 & 5 & 3 & 5 & 4 & 5 & 3 \\
\hline 16 & 4 & 4 & 4 & 4 & 4 & 4 & 4 \\
\hline 20 & 4 & 5 & 4 & 5 & 4 & 5 & 4 \\
\hline 25 & 5 & 5 & 5 & 5 & 5 & 5 & 5 \\
\hline
\end{tabular}

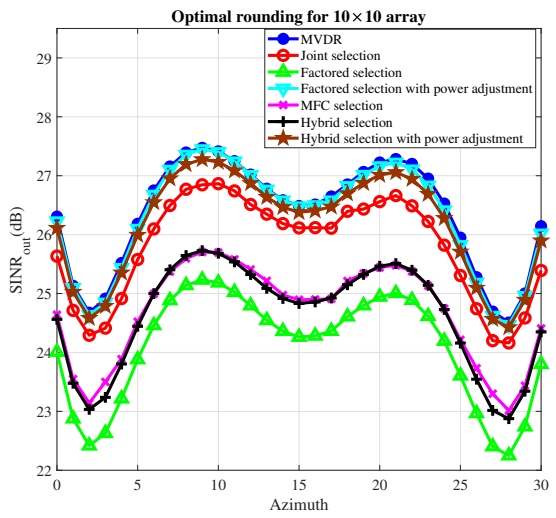

Fig. 7. The optimum SINR out value achieved by the proposed optimization with varying number of selected elements in different selection modes for a $10 \times 10$ array

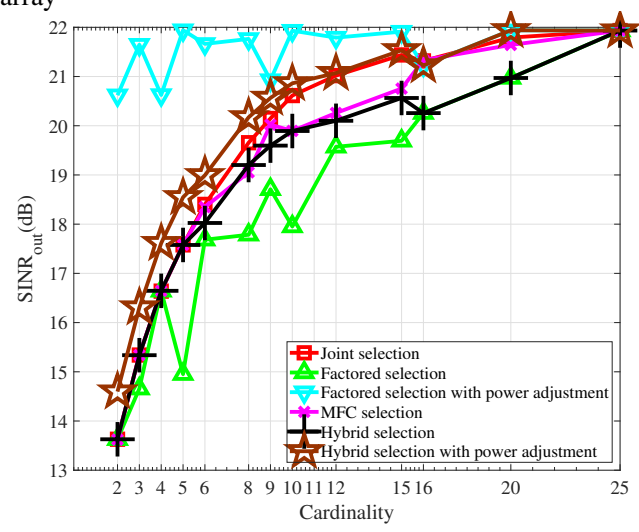

Fig. 8. The optimum SINR out value achieved by the proposed optimization with varying number of selected elements in different selection modes.

maximization problem with quadratic constraints to decouple the problem and optimize three joint vectors separately in a unified problem. Since the selection strategies return the optimal subarrays for the given scenario compared to any arbitrary subarray, the maximum output SINR is always achieved. We then presented a theoretical study demonstrating that the joint selection gives the optimum solution followed by the suboptimal solutions offered by MFC, hybrid and factored modes respectively. Moreover, we proposed an appropriate relaxation and approximation method to tackle the nonconvexity of the primal problem. Finally, we presented extensive simulations that verified the theoretical findings and confirmed the effectiveness of the proposed techniques in reducing the problem dimensionality while maintaining a performance that is comparable to the full array.

\section{APPENDIX A \\ Proof OF THEOREM 1}

Proof: For an arbitrary selection vector we can extend $\mathcal{S}_{1}$ under $\mathcal{S}_{2}$ and $\mathcal{S}_{3}$ as

$\mathbf{c}^{T} \mathbf{Q} \mathbf{c}=\sum_{i=1}^{M+N}\left(\mathbf{c}^{T}[\mathbf{P}]_{i}\right)^{2}=\sum_{i=0}^{k_{\mathrm{t}}-1} \epsilon_{i}\left(k_{\mathrm{t}}-i\right)^{2}+\sum_{i=0}^{k_{\mathrm{r}}-1} \zeta_{i}\left(k_{\mathrm{r}}-i\right)^{2}$,

where $[\mathbf{P}]_{i}$ denotes the $i$-th column of $\mathbf{P}$, and $\epsilon_{i}, \zeta_{i} \in \mathbb{Z}^{*}$, are appropriate integers equal to the number of times the corresponding squared value appears. Here $\mathbb{Z}^{*}$ denotes the set of non-negative integers.

Now, we can establish a set of equations based on $\mathcal{S}_{1}$ to $\mathcal{S}_{4}$ and apply 21) as follows

$$
\begin{aligned}
& \mathcal{S}_{1}: \quad \epsilon_{0} k_{\mathrm{t}}^{2}+\sum_{i=1}^{k_{\mathrm{t}}-1} \epsilon_{i}\left(k_{\mathrm{t}}-i\right)^{2}+\zeta_{0} k_{\mathrm{r}}^{2} \\
& +\sum_{i=1}^{k_{\mathrm{r}}-1} \zeta_{i}\left(k_{\mathrm{r}}-i\right)^{2}=k_{\mathrm{r}} k_{\mathrm{t}}^{2}+k_{\mathrm{t}} k_{\mathrm{r}}^{2} \\
& \mathcal{S}_{2}: \quad \epsilon_{i} \leq k_{\mathrm{r}} \quad i=0, \ldots, k_{\mathrm{t}}-1 \\
& \mathcal{S}_{3}: \quad \zeta_{i} \leq k_{\mathrm{t}} \quad i=0, \ldots, k_{\mathrm{r}}-1 \\
& \mathcal{S}_{4}: \quad \sum_{i=0}^{k_{\mathrm{t}}-1} \epsilon_{i}\left(k_{\mathrm{t}}-i\right)=k_{\mathrm{t}} k_{\mathrm{r}} \\
& \mathcal{S}_{4}: \sum_{i=0}^{k_{\mathrm{r}}-1} \zeta_{i}\left(k_{\mathrm{r}}-i\right)=k_{\mathrm{t}} k_{\mathrm{r}} .
\end{aligned}
$$

Given the above set of equations, we need to show that the only non-trivial solution is

$$
\begin{aligned}
& \epsilon_{0}=k_{\mathrm{r}} \text { and } \epsilon_{i}=0 \text { for } i=1, \ldots, k_{\mathrm{t}}-1 \\
& \zeta_{0}=k_{\mathrm{t}} \text { and } \zeta_{i}=0 \text { for } i=1, \ldots, k_{\mathrm{r}}-1 .
\end{aligned}
$$

Based on 267 and 27) we can write

$$
\epsilon_{0}=k_{\mathrm{r}}-\sum_{i=1}^{k_{\mathrm{t}}-1} \epsilon_{i} \frac{\left(k_{\mathrm{t}}-i\right)}{k_{\mathrm{t}}}, \quad \zeta_{0}=k_{\mathrm{t}}-\sum_{i=1}^{k_{\mathrm{r}}-1} \zeta_{i} \frac{\left(k_{\mathrm{r}}-i\right)}{k_{\mathrm{r}}} .
$$

Then, we extend (22) as

$$
\begin{aligned}
& \left(k_{\mathrm{r}}-\sum_{i=1}^{k_{\mathrm{t}}-1} \epsilon_{i} \frac{\left(k_{\mathrm{t}}-i\right)}{k_{\mathrm{t}}}\right) k_{\mathrm{t}}^{2}+\sum_{i=1}^{k_{\mathrm{t}}-1} \epsilon_{i}\left(k_{\mathrm{t}}-i\right)^{2} \\
& +\left(k_{\mathrm{t}}-\sum_{i=1}^{k_{\mathrm{r}}-1} \zeta_{i} \frac{\left(k_{\mathrm{r}}-i\right)}{k_{\mathrm{r}}}\right) k_{\mathrm{r}}^{2}+\sum_{i=1}^{k_{\mathrm{r}}-1} \zeta_{i}\left(k_{\mathrm{r}}-i\right)^{2}=k_{\mathrm{r}} k_{\mathrm{t}}^{2}+k_{\mathrm{t}} k_{\mathrm{r}}^{2} .
\end{aligned}
$$

Hence, we have

$$
\sum_{i=1}^{k_{\mathrm{t}}-1} \epsilon_{i} i\left(i-k_{\mathrm{t}}\right)+\sum_{i=1}^{k_{\mathrm{r}}-1} \zeta_{i} i\left(i-k_{\mathrm{r}}\right)=0 .
$$


Noting that

$$
\epsilon_{i} \geq 0, \quad \zeta_{i} \geq 0, \quad i \geq 1, \quad\left(i-k_{\mathrm{t}}\right)<0, \quad\left(i-k_{\mathrm{r}}\right)<0,
$$

It follows easily that (28) holds if and only if

$$
\epsilon_{i}=0 \text { for } i=1, \ldots, k_{\mathrm{t}}-1, \quad \zeta_{i}=0 \text { for } i=1, \ldots, k_{\mathrm{r}}-1,
$$

and accordingly

$$
\epsilon_{0}=k_{\mathrm{r}} \quad, \quad \zeta_{0}=k_{\mathrm{t}} .
$$

\section{APPENDIX B}

\section{PROOF OF THEOREM 4}

Proof: We introduce the quadratic function $e(\mathbf{y}), \mathbf{y} \in R^{k_{t}}$

as

$$
e(\mathbf{y})=\mathbf{c}^{T} \mathbf{Q}_{\mathrm{t}} \mathbf{c}=\mathbf{c}^{T} \mathbf{P}_{\mathrm{t}} \mathbf{P}_{\mathrm{t}}^{T} \mathbf{c}=\sum_{i=1}^{M}\left(\mathbf{c}^{T}\left[\mathbf{P}_{\mathrm{t}}\right]_{i}\right)^{2}=\sum_{i=1}^{k_{\mathrm{t}}} y_{i}^{2} .
$$

To find the lower-bound, we solve the following minimization

$$
\begin{array}{ll}
\min _{\mathbf{y}} & e(\mathbf{y}) \\
\text { s.t. } & \mathbf{1}_{k_{\mathrm{t}}}^{T} \mathbf{y}=k_{\mathrm{r}} k_{\mathrm{m}} .
\end{array}
$$

Solving this problem using the Lagrangian method yields

$$
y_{i}=\frac{k_{\mathrm{r}} k_{\mathrm{m}}}{k_{\mathrm{t}}}, \quad i=1, \ldots, k_{\mathrm{t}}
$$

and subsequently the lower-bound is given by

$$
e(\mathbf{y})=\frac{\left(k_{\mathrm{r}} k_{\mathrm{m}}\right)^{2}}{k_{\mathrm{t}}}
$$

The upper-bound is achieved by letting $k_{\mathrm{m}}=k_{\mathrm{t}}$ yielding

$$
e(\mathbf{y})=k_{\mathrm{r}}^{2} k_{\mathrm{m}}
$$

\section{REFERENCES}

[1] E. Larsson, O. Edfors, F. Tufvesson, and T. Marzetta, "Massive MIMO for next generation wireless systems," IEEE Commun. Mag., vol. 52, no. 2, pp. 186-195, 22014.

[2] J. Mietzner, R. Schober, L. Lampe, W. Gerstacker, and P. Hoeher, "Multiple-antenna techniques for wireless communications - a comprehensive literature survey," IEEE Communications Surveys \& Tutorials, vol. 11, no. 2, pp. 87-105, 2009.

[3] J. Li and P. Stoica, "MIMO Radar Diversity Means Superiority," in MIMO Radar Signal Processing. Hoboken, NJ, USA: John Wiley \& Sons, Inc., ch. 1, pp. 1-64.

[4] A. Hassanien and S. A. Vorobyov, "Why the phased-mimo radar outperforms the phased-array and MIMO radars," in European Signal Processing Conference, vol. 58, no. 6, 6 2010, pp. 1234-1238.

[5] D. Bliss and K. Forsythe, "Multiple-input multiple-output (MIMO) radar and imaging: degrees of freedom and resolution," Conference Record of the Thirty-Seventh Asilomar Conference on Signals, Systems and Computers, vol. 1, pp. 54-59, 2003.

[6] A. F. Molisch and M. Z. Win, "MIMO systems with antenna selection," IEEE Microw. Mag., vol. 5, no. 1, pp. 46-56, 2004.

[7] R. Heath, S. Sandhu, and A. Paulraj, "Antenna selection for spatial multiplexing systems with linear receivers," IEEE Commun. Lett., vol. 5, no. 4, pp. 142-144, apr 2001.

[8] X. Wang, E. Aboutanios, M. Trinkle, and M. G. Amin, "Reconfigurable Adaptive Array Beamforming by Antenna Selection," IEEE Trans. Signal Process., vol. 62, no. 9, pp. 2385-2396, 52014.
[9] X. Wang, M. Amin, and X. Cao, "Analysis and Design of Optimum Sparse Array Configurations for Adaptive Beamforming," IEEE Trans. Signal Process., vol. 66, no. 2, pp. 1-1, 2017.

[10] M. Gharavi-Alkhansari and A. B. Gershman, "Fast antenna subset selection in MIMO systems," IEEE Trans. Signal Process., vol. 52, no. 2, pp. 339-347, 2004.

[11] A. Gorokhov, "Antenna selection algorithms for MEA transmission systems," IEEE International Conference on Acoustics Speech and Signal Processing, no. 2, pp. III-III, 2002.

[12] I. Berenguer and V. Krishnamurthy, "Adaptive MIMO antenna selection via discrete stochastic optimization," IEEE Trans. Signal Process., vol. 53, no. 11, pp. 4315-4329, 2005.

[13] R. Mendez-Rial, C. Rusu, N. Gonzalez-Prelcic, A. Alkhateeb, and R. W. Heath, "Hybrid MIMO Architectures for Millimeter Wave Communications: Phase Shifters or Switches?" IEEE Access, vol. 4, pp. 247-267, 2016.

[14] Y. Liu, Q. H. Liu, and Z. Nie, "Reducing the number of elements in multiple-pattern linear arrays by the extended matrix pencil methods," IEEE Trans. Antennas Propag., vol. 62, no. 2, pp. 652-660, feb 2014.

[15] W. P. M. N. Keizer, "Linear array thinning using iterative FFT techniques," IEEE Trans. Antennas Propag., vol. 56, no. 8, pp. 2757-2760, aug 2008 .

[16] X. Wang, E. Aboutanios, and M. G. Amin, "Adaptive array thinning for enhanced DOA estimation," IEEE Signal Process. Lett., vol. 22, no. 7, pp. 799-803, July 2015.

[17] A. Deligiannis, M. Amin, S. Lambotharan, and G. Fabrizio, "Optimum sparse subarray design for multitask receivers," IEEE Trans. Aerosp. Electron. Syst., pp. 1-1, 2018.

[18] H. Nosrati, E. Aboutanios, and D. Smith, "Array partitioning for multitask operation in dual function MIMO systems," Digital Signal Process. vol. 82, pp. 106-117, nov 2018.

[19] Q. He, R. S. Blum, H. Godrich, and A. M. Haimovich, "Target velocity estimation and antenna placement for MIMO radar with widely separated antennas," IEEE J. Sel. Topics Signal Process., vol. 4, no. 1, pp. $79-100$, feb 2010

[20] H. Godrich, A. P. Petropulu, and H. V. Poor, "Sensor selection in distributed multiple-radar architectures for localization: A knapsack problem formulation," IEEE Trans. Signal Process., vol. 60, no. 1, pp. 247-260, jan 2012.

[21] A. A. Gorji, R. Tharmarasa, and T. Kirubarajan, "Optimal antenna allocation in MIMO radars with collocated antennas," IEEE Trans. Aerosp. Electron. Syst., vol. 50, no. 1, pp. 542-558, jan 2014.

[22] B. Ma, H. Chen, B. Sun, and H. Xiao, "A joint scheme of antenna selection and power allocation for localization in MIMO radar sensor networks," IEEE Commun. Lett., vol. 18, no. 12, pp. 2225-2228, dec 2014.

[23] C. Y. Chen and P. P. Vaidyanathan, "Minimum redundancy MIMO radars," in Proceedings - IEEE International Symposium on Circuits and Systems. IEEE, 5 2008, pp. 45-48.

[24] J. Dong, Q. Li, and W. Guo, "A combinatorial method for antenna array design in minimum redundancy MIMO radars," IEEE Antennas Wireless Propag. Lett., vol. 8, pp. 1150-1153, 2009.

[25] Y. Li, S. A. Vorobyov, and A. Hassanien, "MIMO radar capability on powerful jammers suppression," in 2014 IEEE International Conference on Acoustics, Speech and Signal Processing (ICASSP). IEEE, may 2014.

[26] P. P. Vaidyanathan and P. Pal, "MIMO radar, SIMO radar, and IFIR radar: a comparison," in 2009 Conference Record of the Forty-Third Asilomar Conference on Signals, Systems and Computers. IEEE, 2009.

[27] H. L. Van Trees, Optimum Array Processing, 2002, vol. Part IV, no. S1.

[28] X. Wang, E. Aboutanios, and M. G. Amin, "Slow radar target detection in heterogeneous clutter using thinned space-time adaptive processing," IET Radar, Sonar \& Navigation, vol. 10, no. 4, pp. 726-734, apr 2016.

[29] S. Boyd and L. Vandenberghe, Convex optimization. Cambridge University Press, 2004.

[30] S. Joshi and S. Boyd, "Sensor selection via convex optimization," IEEE Trans. Signal Process., vol. 57, no. 2, pp. 451-462, 2009.

[31] H. Nosrati, E. Aboutanios, and D. Smith, "Receiver-Transmitter Pair Selection in MIMO Phased Array Radar," The 42nd IEEE International Conference on Acoustics, Speech and Signal Processing, pp. 3206-3210, 2017.

[32] H. Nosrati, E. Aboutanios, and D. B. Smith, "Matched filter constrained MIMO array spatial thinning for interference mitigation," in 2017 IEEE Radar Conference (RadarConf). IEEE, may 2017.

[33] S. Boyd, l1-norm methods for convex cardinality problems. Lecture Notes for EE364b, Stanford University. Available at http://www. stanford. edu/class/ee364b, 2007. 
[34] Y. Nesterov and A. Nemirovskii, Interior-Point Polynomial Algorithms in Convex Programming. Society for Industrial and Applied Mathematics, jan 1994.

[35] T. Lipp and S. Boyd, "Variations and extension of the convex-concave procedure," Optimization and Engineering, vol. 17, no. 2, pp. 263-287, nov 2015.

[36] M. Grant, S. Boyd, and Y. Ye, "CVX: Matlab software for disciplined convex programming," 2008. 